University of Louisville

ThinkIR: The University of Louisville's Institutional Repository

Electronic Theses and Dissertations

8-2007

\title{
Development of ground penetrating radar image library for setup parameters.
}

Claudel Nisingizwe 1980-

University of Louisville

Follow this and additional works at: https://ir.library.louisville.edu/etd

\section{Recommended Citation}

Nisingizwe, Claudel 1980-, "Development of ground penetrating radar image library for setup parameters." (2007). Electronic Theses and Dissertations. Paper 1064.

https://doi.org/10.18297/etd/1064

This Master's Thesis is brought to you for free and open access by ThinkIR: The University of Louisville's Institutional Repository. It has been accepted for inclusion in Electronic Theses and Dissertations by an authorized administrator of ThinkIR: The University of Louisville's Institutional Repository. This title appears here courtesy of the author, who has retained all other copyrights. For more information, please contact thinkir@louisville.edu. 


\title{
DEVELOPMENT OF GROUND PENETRATING RADAR
} IMAGE LIBRARY FOR SETUP PARAMETERS

\author{
By
}

\section{Claudel Nisingizwe}

B. Sc. Civil Engineering and Environmental Technology KIST Rwanda, 2004

A Thesis Submitted to the Faculty of the Graduate School of the University of Louisville in Partial Fulfillment of the Requirement For The Degree of

\section{MASTER OF SCIENCE}

Department of Civil and Environmental Engineering, University of Louisville

Louisville, Kentucky 


\title{
DEVELOPMENT OF GROUND PENETRATING RADAR IMAGE \\ LIBRARY FOR PARAMETER SETUP COMPARISON
}

\author{
By
}

\section{Claudel Nisingizwe}

B.Sc. Civil Engineering and Environmental Technology

KIST Rwanda, 2004

A Thesis Approved on

May 9, 2007

By the following thesis committee:

Dr. J. P. Mohsen, Thesis Director

Dr. T. D. Rockaway

Dr. A. H. Desoky 


\title{
DEDICATION
}

This thesis is dedicated

To my parents

Onesphore Rwaje and Josephine Mujawiyera

\author{
To my sisters \\ Josiane Manishimwe and Claudine Nishimwe
}

\begin{abstract}
And my brothers
Josue Niyonsingiza and Jonathan Duchimimana
\end{abstract}

Who have always supported and encouraged me. 


\section{ACKNOWLEDGEMENTS}

I would like to express my deep appreciation to my advisor Dr. J. P. Mohsen, for his support, guidance and encouragement during this experiments and the past year. I would also like to thank my committee members, Dr. Thomas D. Rockaway and Dr. Ahmed H. Desoky.

I would like to extend sincere gratitude to Dr. M. French, Dr. D. J. Hagerty, Dr. C. Robert Ullrich, Dr. Nageshwar R. Baskar, Dr. Terry A. Weigel, Mr. Bernie Miles, Ms. Leanne Whitney and Ms. Gail Grave for their encouragement and help throughout my study and experiments .

I also would like to give special thanks to my family and friends for their encouragement during my studies. 


\begin{abstract}
Development of Ground Penetrating Radar Image Library for Parameter Setup Comparison
\end{abstract}

\title{
Claudel Nisingizwe
}

May 09, 2007

A significant amount of effort has been put in developing tools to interpret Ground Penetrating Radar signals obtained during surveys. Currently, skilled and experienced users do most of GPR image interpretation. They use experience in deciphering what the GPR signals represent.

A successful survey will not only depend on the choice of antenna used, but also on the operating parameters used for the survey. This study aims at providing a library of GPR images taken from known targets with 
known parameters. The targets include a set of different sizes of steel rebars.

The library of GPR images is developed using known targets set in a sand box. Sand has proven to have the same properties as Portland cement concrete in response to GPR signals. The sand box simulates a concrete slab; it is used for ease of placement of different targets with various configurations.

The developed library of GPR images will be used for training of GPR users and comparison studies of GPR operating parameters. In the future these images can be used for a pattern recognition algorithm development, or any other theoretical study pertaining to GPR image interpretation. 
TABLE OF CONTENTS

PAGE

ACKNOWLEDGEMENT iv

ABSTRACT

TABLE OF CONTENTS

$\mathrm{V}$

LIST OF TABLES

vii

LIST OF FIGURES

CHAP.I INTRODUCTION

I.1 BACKGROUND OF THE RESEARCH

I.2 SIGNIFICANCE OF THE RESEARCH ............

I.3 OBJECTIVES OF THE RESEARCH …...............

I.4 SCOPE OF THE RESEARCH …….......................

CHAP.II LITERATURE REVIEW .............................................

II. 1 SIMILAR EXPERIMENT SETUP …....................

II.2 PATTERN RECOGNITION ....................................

II. 3 GPR APPLICATION FOR URBAN AREAS ...

II.4 TEST SITES ........................................................... 
III.2 CLASSIFICATION OF NDT METHODS

III.3 NDT METHODS FOR CONCRETE

EVALUATION

1. STRENGTH OF CONCRETE STRUCTURE TEST METHODS.

2. FEATURE AND CONCRETE

CHAP.IV GPR TECHNOLOGY

1. ELECTROMAGNETIC ENERGY

2. WAVELENGTH

3. FREQUENCY

4. PERIOD

5. ELECTROMAGNETIC SPECTRUM

6. RADAR ENERGY 
1. ANTENNA

2. ELECTRICAL CONDUCTIVITY

3. DIELECTRIC CONSTANT

4. MATERIAL DIELECTRIC CONSTANT DETERMINATION

5. DETECTION METHODS

CHAP.V EQUIPMENT

V.I COMPONENTS OF THE EQUIPEMENT ...............

1. CONTROL UNIT

2. POWER SUPPLY

3. ANTENNA

4. SURVEY WHEEL

V.2 EQUIPMENT OPERATION

1. HARDWARE SETUP

2. DATA ACQUISITION

3. PARAMETER SETUP

4. DATA DISPLAY

5. DATA PROCESSING 
1. PARAMETERS THAT CAN BE CUSTOMIZED

VII. 2 DEPTH PARAMETER EFFECTS ............

VII.4 STEEL REBAR SIZE

VII.5 ONE STEEL REBAR VS TWO STEEL REBARS.

VII. 6

HORIZONTAL DISTANCE

1. EQUAL SIZE STEEL REBARS

2. DIFFERENT SIZE STEEL REBARS

3. SCAN SPACING EFFECTS

4. DEPTH OF STEEL REBAR EFFECTS

1. EQUAL SIZE STEEL REBARS 


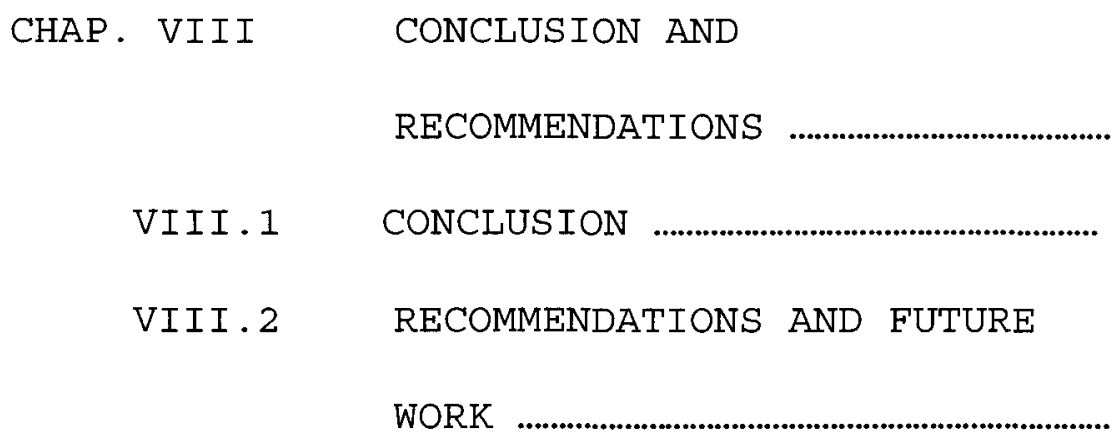




\section{LIST OF TABLES}

1 Nondestructive testing method categories ... 20

2 Electromagnetic spectrum table .................................. 40

3 Typical range values for different GPR antenna frequencies .................................................................

4 Dielectric constant for some common materials

5 Processing objectives and methods

7 Data display mode 


\section{LIST OF FIGURES}

\section{FIGURE}

1 Image of a masonry wall

2 Image of a masonry wall under infrared scanning

3 Schematic diagram of a rolling dynamic deflectometer

4 Stationary transient load system

5 Equipment image of Pulse echo system ............

6 Image of the equipment

7 Linescan format sample

8 Wiggle format sample

9 O-scope format sample

10 Line scan with wiggle format sample

11 3D display format sample

12 Sand box

13 A steel rebar GPR signal

14 Scan spacing effects of a No 9 steel rebar, 3" deep

15 Depth parameter effects of a No 9 steel 
16 GPR signals of a No 9 steel rebar at different depths

17 GPR signals of a No 3, No 4, No 6, No 7, No 8 , No 9 steel rebars, 3 " deep.

18 GPR signals of one No 8 steel rebar and two No 8 steel rebars placed next to each other

19 Horizontal distance effects between two No 8 steel rebars, $1^{\prime \prime}$ deep

20 Close up of the parabola peaks of two No 8 at $0^{\prime \prime}, 1^{\prime \prime}, 2 "$ apart

21 Horizontal distance effects between a No 3 and No 9 steel rebars

22 Close up of the parabola peaks of a No 3 and No 9 next to each other

23 Scan spacing effects on GPR signals of two No 8 steel rebars, $1^{\prime \prime}$ deep and 1" apart

24 Effect of depth of placement on visibility of two No 8 steel rebars, different placement depths

25 Vertical distance effects between two No 8 steel rebars

26 Vertical distance effects between a No 3 and No 9 steel rebars 


\section{CHAP.I INTRODUCTION}

Ground Penetrating Radar (GPR) is used as a Non-

destructive tool for maintenance (e.g. inspection of roads, airports runways, bridges, architectural

facades...) and development applications such as detection of existing underground utility pipes. Other applications include detecting minerals, mining, ice thickness measurement, archaeology and forensic investigation.

Interpretation of GPR images is difficult. The difficulty level depends on the complexities of the environment being surveyed and the amount of noise found in the images. A database or library that catalogs the different parameters and GPR signal responses to various field conditions and various operating parameters will be of significant help in increasing the experience of GPR practitioners. 
A library of images with changes of operating parameters and feature configurations is presented. This library is developed by using GPR images developed from a sand box. Different target configurations were made in order to assess the GPR antenna capabilities in detecting them; the same configurations were surveyed with different operating parameter settings in order to assess the impact of these parameters on the signals received.

\section{1 BACKGROUND OF THE RESEARCH}

A project to construct a test field that models common urban environment conditions is under way at the University of Louisville, Louisville, Kentucky. It is intended that this field will serve as a research and testing facility for both academics and practitioners active in GPR and other NDT techniques.

The University of Louisville test field is designed and will be constructed modeling the conditions of a street intersection in an urban area with normal utility features encountered in an urban setting. It 
will be used to assess and develop new GPR analysis procedures in controlled environments. Part of the test field will also feature an array of different buried materials.

Objectives of this project include:

- A test field to be used for calibration and training.

- Incorporated in non destructive testing studies.

- Develop a training and seminar program for new GPR users.

A previous study that focused on interpretation of voids under urban settings was done at the University of Louisville, it was reported by ZHIYONG ZHAO in his dissertation: "Application of Ground Penetrating Radar to Locate Subsurface Voids in Urban Areas". The current study will compliment it.

\section{I.2 SIGNIFICANCE OF THE RESEARCH}

This research is part of the larger scope of the university's project in training GPR practitioners and 
in assessing and developing new GPR analysis and interpretation procedures in controlled environments.

An extensive library of GPR images is developed by cataloging GPR signal images under different configurations and operating parameters settings.

\section{I.3 OBJECTIVES OF THE RESEARCH}

This research is conducted in order to complement the field tests with specific GPR images that can be used towards the introduction and understanding of certain operating parameters of the GPR system and their impacts on collection of data.

In general most of GPR data analysis and NDT methods involve study of collected images. A trained practitioner can recognize patterns and thus make a very good assumption of what the images represent. The objective of these experiments is to provide GPR images from known targets, under different configurations and operating parameter settings. Thus assist in broadening the practitioners' experience. 


\section{I.4 SCOPE OF THE RESEARCH}

The GPR antenna used has a frequency $1.5 \mathrm{GHz}$. A sand box was used to simulate concrete structures.

The experiments are not exhaustive in that all possible configurations were not made; all possible material types and sizes were not used. All operating parameters were not studied only those that pertain to the Concretescan mode were studied.

The scope of the thesis includes a summary of NDT methods in their evaluation of concrete and subsurface evaluation, a study of the principle of electromagnetism and GPR principle and the experiments conducted are described and analyzed. At last, the conclusion, recommendations and proposed future work are provided. 


\section{CHAP.II LITERATURE REVIEW}

Detecting objects buried beneath the Earth's surface or situated interior to a visually opaque medium has been a topic of interest to many researchers across different disciplines. A number of GPR algorithms have been adopted for various applications from mine detection to geophysics (Caner Ozdemir and Hao Ling; June 2006).

It is often necessary to identify the location or assess the integrity of buried infrastructure within an urban environment. It is difficult to achieve that goal with NDT methods due to the existence of heterogeneities within this environment (different infrastructure materials, different infrastructure types, different surface materials). 
This chapter will present work done at other institution that shares certain similarities with the current project reported.

\section{II.1 SIMILAR EXPERIMENT SETUP}

In 2005, Caner Ozdemir and Hao Ling presented a paper: "An Experiment Investigation of Buried Object Imaging in a Homogeneous Medium using Synthetic Aperture Radar Concepts". In this paper, they presented a similar approach to the one that was used in burying water and plastic bottles in sand and producing their images. Their objective was to apply a Fourier based imaging algorithm based on synthetic aperture radar (SAR) concepts, to the signals received. They developed and presented the algorithm which can successfully be used in detecting high-contrast and low-contrast objects.

\section{II.2 PATTERN RECOGNITION}

Melten Ballan and Filkret Gurgen, in 2000 proposed a classification method for $2 \mathrm{D}$ images in their paper: "2D GPR Data Identification"; their classification method was based on non-parametric approximation to 
Bayes decision probability. It was a pattern recognition based feature extraction and classification method. Storage required for the data was large and this presented a disadvantage. Their classification method was done after investigating the output, and in general they worked with pre-processed data.

Richard Yelf and Waleed Al-Nuaimy, in their paper, "classification System for GPR Parameters" 2000, proposed a classification system used to classify large GPR data sets for civil engineering and geotechnical applications. The system uses two sets of numerical descriptors to code the interpreted ground conditions. They used two parameters namely the GPR class which addresses the relative excavation capability of the soil and the GPR Rock Type Descriptors which describes the soil/rock type and also the attributes of the material condition.

In the paper "Investigation of the GPR reflection pattern for shallow depths on a test site" by Rolf Gerber, Peter Felix-Henningsen, Christina Salat and Andreas Junge reported on their study of different 
materials with a varying degree of weathering as well as an artificial reflector. Investigations of the reflection patterns were also performed with respect to the antenna orientation, dielectric coefficient, attenuation, soil water content and the corresponding reflection coefficients of the different materials.

\section{3 GPR APPLICATION FOR URBAN AREAS}

GPR has been proposed as an efficient tool in detecting underground utilities especially in urban areas. In Europe, a project named the European Giga project uses GPR technology in mapping the underground in order to develop well controlled and reliable gas networks, as well as telecommunication links. Guido Manacorda, Howard Scott, Paul D Loach, John J Kazik, Dave Pinchbeck, Meinolf Remeil, Jean Pierre Capdevielle, Pascal Fabien Fournier; "The Europian GIGA project" 2004 .

Multichannel imaging radar systems have been found to be effective for the underground utility mapping to single channel antenna GPR systems as they provide images from different antennas with different 
frequencies. Egiil S. Eide and Jens F. Hjelmstad "3D Utility mapping Using Electronically Scanned Antenna Array" 2001. Ralf Birken, Douglas E. miller, Maclyn Burns, Paul Albats, Robert Casadonte, Ross Deming, Tony Derubeis, Thorkild Hansen and Micheal Oristaglio "Efficient Large-Scale Underground Utility Mapping in New York City Using a Multi Channel GPR Imaging Radar System", 2001

In their paper, Ground Penetrating Radar Investigation for Urban Roads, R. Evans, M. Frost, M. Stonecliffe Jones, presented GPR as a tool for pavement evaluation. They used a multichanel antenna of 1.5 $\mathrm{GHz}, 400 \mathrm{MHz}$ and $100 \mathrm{MHz}$, and were successful in identifying a safe planning depth to which materials could be removed. They were able to identify the bottom of the bituminous layer but were unable to identify lower layers.

\section{4 TEST SITES}

Test sites have been constructed with different objectives, but the common objective was to study the abilities of GPR systems. 
In the paper: "The Measured Effect of Soils on GPR Antenna Resolution" Will Clark, Brian Burns, James Ralston and Elvis Dieguez reported on the effects of different soils on an Antenna response, their test field had three types of soils namely, sand, crushed run gravel and bank run gravel.

In the study reported in the paper "Investigation of the GPR reflection pattern for shallow depths on a test site" by Rolf Gerber, Peter Felix-Henningsen, Christina Salat and Andreas Junge used a test site which contained structures of different geometry within a depth of one meter. The test site was designed to resemble the characteristic layered composition of a basal layer with a high stone content and loess-rich upper layers.

A test site was used with the objective of measuring the penetration of GPR signals and the resolution in distinguishing anomalies of different types of GPR antennas. The test site was filled with 3 different types of soils, sandy soil, shale and granitic soil; it contained a distribution of manufactured elements 
of various materials and sizes. Jesus Paniagua, Mariano del Rio and Montana Rufo "Test Site for the Analysis of Subsoil GPR Signal Propagation".

Test fields in Ohio were used to study the capabilities of GPR in detecting and locating drainage pipes in farmlands. Barry J. Allred, Norman R. Fauset, Chi-Chih Chen, Leon Peters, Jr. and Hyoung sun Youn "Detection of drainage Pipes in Farmlands" 2004; the data generated were not affected by the fired clay or plastic material of which the pipes were comprised.

\section{II.5 TARGET SIZE INVESTIGATION}

This project will develop a library of raw GPR data images to help GPR users in understanding various GPR operational parameters and to have a quick understanding of what the images generated show.

Various studies have been performed and a number of algorithms designed in order to determine the shape and size of features from GPR signals detected during surveys. 
Angelo Liseno, Rocco Pierri, Raffaele Solimene and Francesco Soldovieri have developed an algorithm that reconstitutes the shapes of buried objects and cavities. The algorithm is based on a number of hypotheses such as cylinders that have smooth closed boundaries and do not cast shadows on one another. They presented their findings in their paper: "Shape Reconstitution Algorithm for Buried objects and Cavities" 2004. Another algorithm was developed by Jing Li, Huichun Xing, Xuemin Chen, Y. Sun, Richard Liu, Hua Chen, ED Oshinski, Moon Won and German Claros; it was presented in their paper "Extracting Rebar Reflection from Measured GPR Data"; The Algorithm uses 2 dimensional GPR data. It establishes a set of functions, the variables of which include rebar depths, rebar spacing, pavement thickness and electrical parameters, GPR transmitted waveform and rebar's reflection fields. They proposed an algorithm that not only extracts the rebar's reflection fields but also solves the other pavement parameters. Their measured tolerance was less than $5 \%$

In the paper: "Buried Target Signature Extraction from Ground Penetrating Radar Signal Based on the 
Equivalence Principle", Van den Bosch I., Vander Vorst A. and Lambot $S$. presented a method to subtract the soil contribution from the total GPR signals. The images received are therefore the buried objects signature. This method is based on the equivalence principle.

\section{6 RADIUS DETERMINATION STUDIES}

Estimation of subsurface cylindrical objects radii is done, using an equation for hyperbolae that applies a curve fitting technique after a series of image processing. This technique was found to estimate the depth and radius of the objects to within $1 \%$. The equation models hyperbolic signatures that resulted from the buried cylindrical objects. It takes into account the effect of depth and the relative permittivity of the medium used. "Radius Estimation For Subsurface Cylindrical Objects Detected by Ground Penetrating Radar" S.Shihab, W. Al-Nuamy and A. Eriksen; (2004).

In their paper: "Optimal Radius Estimation for Subsurface Pipes Detected by Ground Penetrating Radar" 
(2006) Vladimir Zolotarev, Andrey Dolgiy, Anatoly

Dolgiy, presented a study done to determine the optimal technique for practical application of GPR to radius estimation of buried pipes. They used techniques based on the weighted least square method, the recursive Kalman filter, the maximum likelihood method, the direct least-square fitting of hyperbola and the Nelder Mead direct search method of optimization. After a comparative study, they concluded that the recursive Kalman filter technique is the most expedient for practical application though it is not necessarily the most expedient at all pipes radii.

S. Shihab and W. Al-Nuaimy in their papers "Shape Descriptor for Hyperbolic GPR signature Discrimination" and "Hyperbola Fitter for Characterization of Cylindrical Targets in GPR Data"; presented a technique for the automatic segmentation of hyperbolic GPR signatures. Targets were first identified then hyperbolic reflection envelopes were highlighted and reported. This technique was proven to be efficient in forming a robust automatic tool for data segmentation as a preliminary step in a 
comprehensive system for automated interpretation of radar data. A fitting equation that models the hyperbolic signatures that take into account the effects of the radius, the vertical inclination and horizontal orientation of the buried cylinders. The new method and fitter showed improved accuracy and reliability. 


\title{
CHAP.III NON-DESTRUCTIVE TESTING LITERATURE
}

\author{
III.1 INTRODUCTION
}

Nondestructive testing (NDT) is a form of testing that does not destroy the tested object. NDT methods are vital for the construction and maintenance of all types of components and structures.

\section{III.2 CLASSIFICATION OF NDT METHODS}

The classification herein presented follows the ASNT classification of NDT methods.

ASNT: American Society of Nondestructive Testing

NDT methods are classified into six major categories: visual, penetrating radiation, magnetic-electrical, mechanical vibration, thermal and chemicalelectrochemical. 
Table III 1 presents the classification system, with additional categories included to cover new methods. The first six categories involve physical processes that require transfer of matter or energy to the object being tested. Two auxiliary categories describe processes that provide for transfer and accumulation of information and evaluation of the raw signals and images common to nondestructive testing methods.

\begin{tabular}{|l|l|}
\hline Basic & Objectives \\
Mechanical and & color, cracks, dimensions, film \\
thickness, reflectivity, strain \\
distribution and magnitude, surface \\
finish, surface flaws, through-cracks \\
Penetrating & $\begin{array}{l}\text { cracks, density and chemistry } \\
\text { variations, elemental distribution, } \\
\text { foreign objects, inclusions, micro- } \\
\text { porosity, misalignment, missing } \\
\text { parts, segregation, service } \\
\text { degradation, shrinkage, thickness, } \\
\text { voids } \\
\text { Alloy content, anisotropy, cavities, }\end{array}$ \\
\hline
\end{tabular}




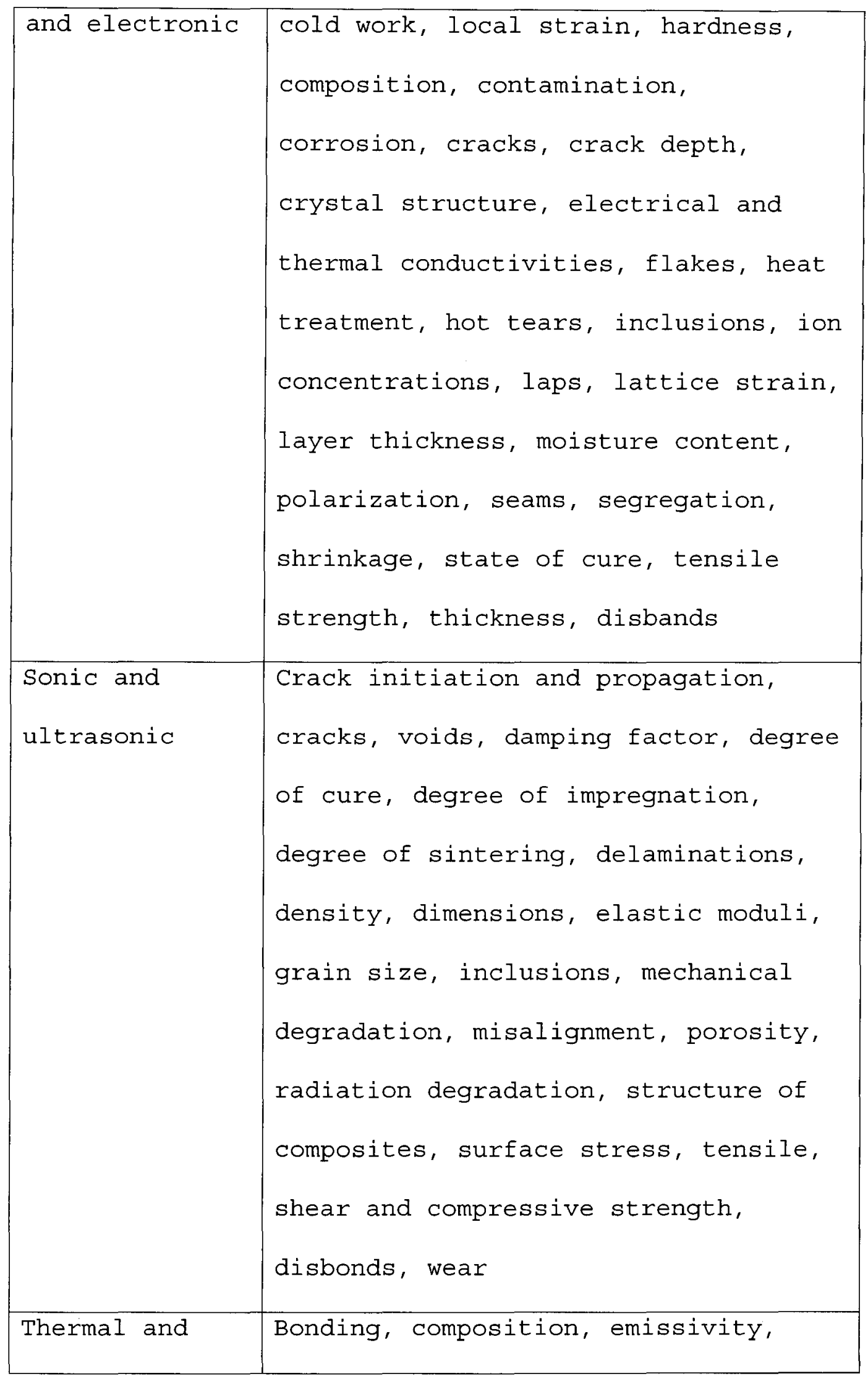




\begin{tabular}{|c|c|}
\hline infrared & $\begin{array}{l}\text { heat contours, plating thickness, } \\
\text { porosity, reflectivity, stress, } \\
\text { thermal conductivity, thickness, } \\
\text { voids }\end{array}$ \\
\hline $\begin{array}{l}\text { Chemical and } \\
\text { analytical }\end{array}$ & $\begin{array}{l}\text { Alloy identification, composition, } \\
\text { cracks, elemental analysis and } \\
\text { distribution, grain size, inclusions, } \\
\text { macrostructure, porosity, } \\
\text { segregation, surface anomalies }\end{array}$ \\
\hline $\begin{array}{l}\text { Auxiliary } \\
\text { Categories }\end{array}$ & Objectives \\
\hline $\begin{array}{l}\text { Image } \\
\text { generation }\end{array}$ & $\begin{array}{l}\text { Dimensional variations, dynamic } \\
\text { performance, anomaly characterization } \\
\text { and definition, anomaly distribution, } \\
\text { anomaly propagation, magnetic field } \\
\text { configurations }\end{array}$ \\
\hline $\begin{array}{l}\text { Signal image } \\
\text { analysis }\end{array}$ & $\begin{array}{l}\text { Data selection, processing and } \\
\text { display, anomaly mapping, correlation } \\
\text { and identification, image } \\
\text { enhancement, separation of multiple } \\
\text { variables, signature analysis }\end{array}$ \\
\hline
\end{tabular}

Table 1: Nondestructive testing method categories 
Each method can be completely characterized in terms of five principal factors:

- energy source or medium used to probe the test object (such as X-rays, ultrasonic waves or thermal radiation);

- nature of the signals, image or signature resulting from interaction with the test object (attenuation of $\mathrm{X}$-rays or reflection of ultrasound, for example);

- means of detecting or sensing resulting signals (photo emulsion, piezoelectric crystal or inductance coil);

- method of indicating or recording signals (meter deflection, oscilloscope trace or radiograph);

- basis for interpreting the results (direct or indirect indication, qualitative or quantitative, and pertinent dependencies).

The objective of each test method is to provide information about the following material parameters:

- discontinuities (such as cracks, voids, inclusions, delaminations); 
- structure or mal-structure (including crystaline structure, grain size, segregation, misalignment) ;

- dimensions and metrology (thickness, diameter, gap size, discontinuity size);

- physical and mechanical properties (reflectivity, conductivity, elastic modulus, sonic velocity);

- composition and chemical analysis (alloy identification, impurities, elemental distributions);

- stress and dynamic response (residual stress, crack growth, wear, vibration); and

- signature analysis (image content, frequency spectrum, field configuration).

\section{III.3 NDT METHODS FOR CONCRETE EVALUATION}

The normal method of evaluating the quality of concrete in buildings or structures is to test specimens cast simultaneously for compressive, flexural and tensile strengths.

Usually a need arises to know either the location of different features within the concrete slab or below 
the slab while the concrete structure is undisturbed; either for reasons of maintenance or additional construction.

NDT methods to test concrete structures could be subdivided into two broad groups: A group of tests that measures the strength of the concrete structure and a group that has an aim of locating features that constitutes the concrete structure.

\section{STRENGTH OF CONCRETE STRUCTURE TEST METHODS}

The literature of the NDT methods that test strength is mainly taken from the handbook of Non-destructive Testing of Concrete, second edition; edited by V.M. Malhotra and N. J. Carino.

These methods include:

- Surface Hardness Methods: These methods are further divided into two groups: The indentation type, consisting of impacting the surface of the concrete structure with a given mass and measuring the width or depth of the resulting 
indentation. "A correlation is made between the strength of properties and its surface hardness as measured by the indentation methods" (Handbook on Nondestructive Testing of Concrete, second edition; P.1-2.). The second group measures the rebound of a spring-driven hammer and correlates it to the strength of concrete.

- Penetration Resistance method: The hardness or penetration resistance strengths of the concrete structures are measured by relating it to the depth of penetration of probes into concrete.

- Pullout test: The pullout test measures the amount of force required to extract an embedded metal insert with an enlarged head from a concrete specimen or structure. By a previously established relation, the pullout load is used to estimate the compressive strength of concrete.

- Break-off test: The Break-off Test tests the flexural strength of concrete by breaking a test specimen that is obtained from an already constructed structure. The specimen is prepared 
by placing a plastic tube before placing concrete or drilling the concrete.

- The Maturity method: This method is based on measuring concrete temperatures variation during curing of the structure. The data history thus obtained is converted to the strength obtained by maturity functions.

\section{FEATURE AND CONCRETE CONDITIONS TEST METHODS}

The objective of these methods is usually to provide the user an image to be used to visualize internal conditions of concrete structures.

Below is a list of some widely used methods:

- Infrared Thermography: Thermography is the use of an infrared imaging and measurement camera to "see" and "measure" thermal energy emitted from an object. Thermal or infrared energy's wavelength is longer than the wavelengths' range that is visible to the human eye; it's the part 
of the electromagnetic spectrum perceived as heat.

The principle of infrared thermorgraphy is based on the theory that everything with a temperature above absolute zero emits heat. The higher the object's temperature, the greater the IR radiation emitted. Infrared thermography cameras produce images of invisible infrared or "heat" radiation and provide precise non-contact temperature measurement capabilities.

Internal conditions for concrete structures such as pavements, that result in uneven thermal conductivity and thermal capacity conditions can be tested. An example is: Density changes in pavements, voids below the pavements, delaminations, differential moisture content.

The infrared thermography method were found to have many disadvantages, namely the fact that the surface temperature of outdoor objects is altered by many factors including the weather, solar radiation and wind velocity. But the method gives 
a good manifestation of damages to concrete structures. (Phong Luong, Nondestructive damage evaluation of reinforced concrete structure using infrared thermography, Ecole Polytechnique, France; M.R. Clark, D.M. McCann, M.C. Forde: Application of infrared thermograhy to the nondestructive testing of concrete and masonry bridges, University of Edinburgh 2003).

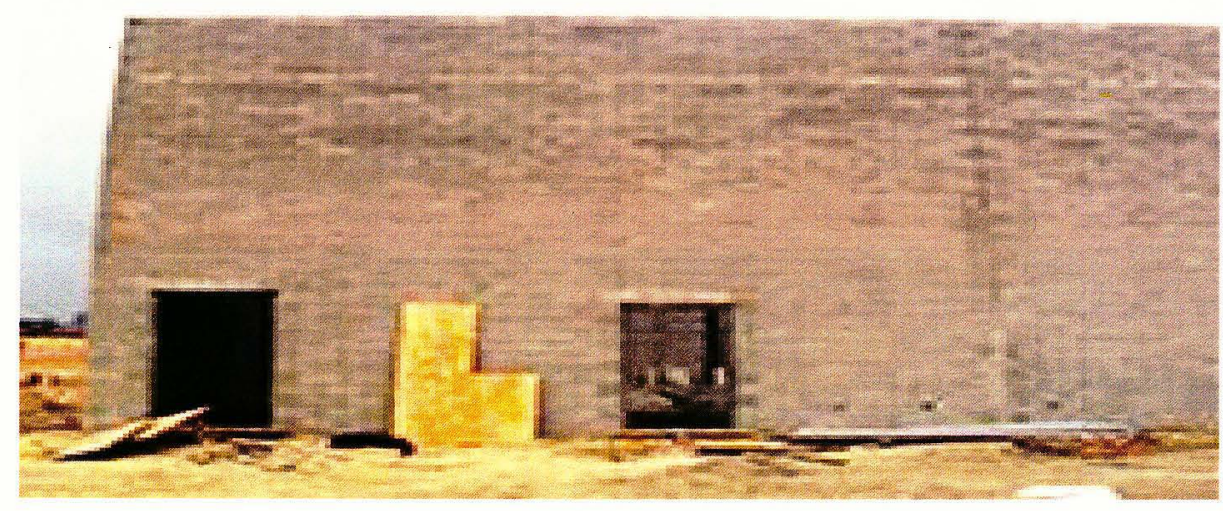

Figure 1: Image of a masonry wall

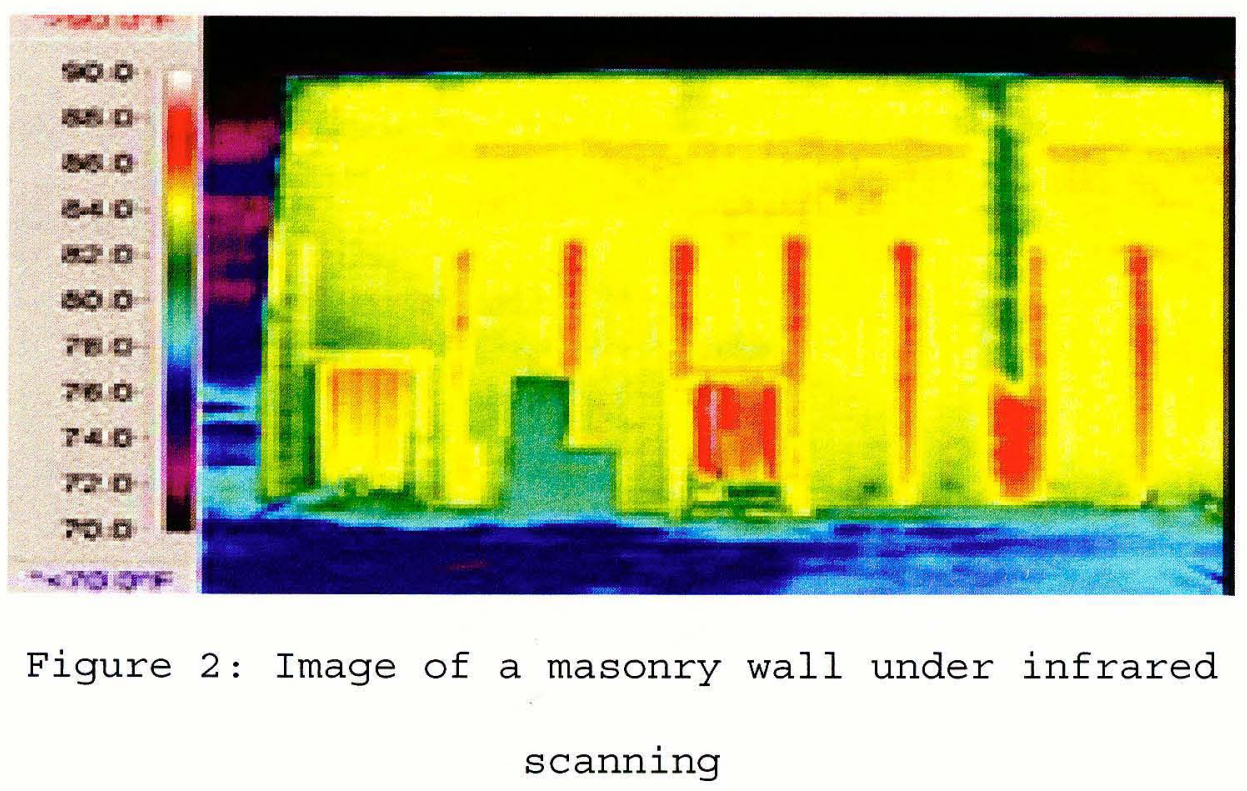


- Ground Penetrating Radar: Ground penetrating radar is a nondestructive geophysical method that produces a continuous cross-sectional profile or record of subsurface features, without drilling, probing, or digging. Ground penetrating radar (GPR) profiles are used for evaluating the location and depth of buried objects and to investigate the presence and continuity of natural subsurface conditions and features.

Ground penetrating radar operates by transmitting pulses of ultra high frequency radio waves (microwave electromagnetic energy) down into the ground through a transducer (also called an antenna). The transmitted energy is reflected from various buried objects or distinct contacts between different earth materials. The antenna then receives the reflected waves and stores them in the digital control unit.

GPR is a great tool for concrete evaluation. It can be used for the determination of concrete deterioration, slab thickness, rebar spacing, bar 
elevation, and amount of concrete cover over the rebar. It is also a great tool to determine the subsoil conditions, it is used to monitor landfills, detect sinkholes and determine the depth of the water table among other uses.

"GPR has two advantages over most other noninvasive geophysical techniques:

1) GPR provides a 3D pseudo-image that can easily be converted to depths that are accurate down to a few centimeters.

2) GPR responds to both metallic and non-metallic objects.

GPR is an excellent tool for mapping nearly any inhomogenity in the subsurface that is characterized by a small difference in density, or porosity." (Jeffrey J. Daniels: GPR fundamentals November 2000).

- Transient load technique: The development of the falling weight deflectometer and the deflectograph are of great importance in evaluating the structural condition of concrete 
pavements. The method measure the surface deflection shape produced from a stationary impulsive load or from a creep rolling wheel load. The deflection measuring equipment using NDT techniques are classified under the following groups :

- Rolling dynamic deflectometer: The Rolling dynamic deflection is used to measure continuous deflection profiles along pavements. (Jeffrey L. Lee and Kenneth H. Stokoe, II December 2005). A truck mounted device is used to apply a large dynamic force to the pavement surface. The resulting deflections are measured by rolling sensors mounted on the truck. The profile created by the deflections is analyzed.

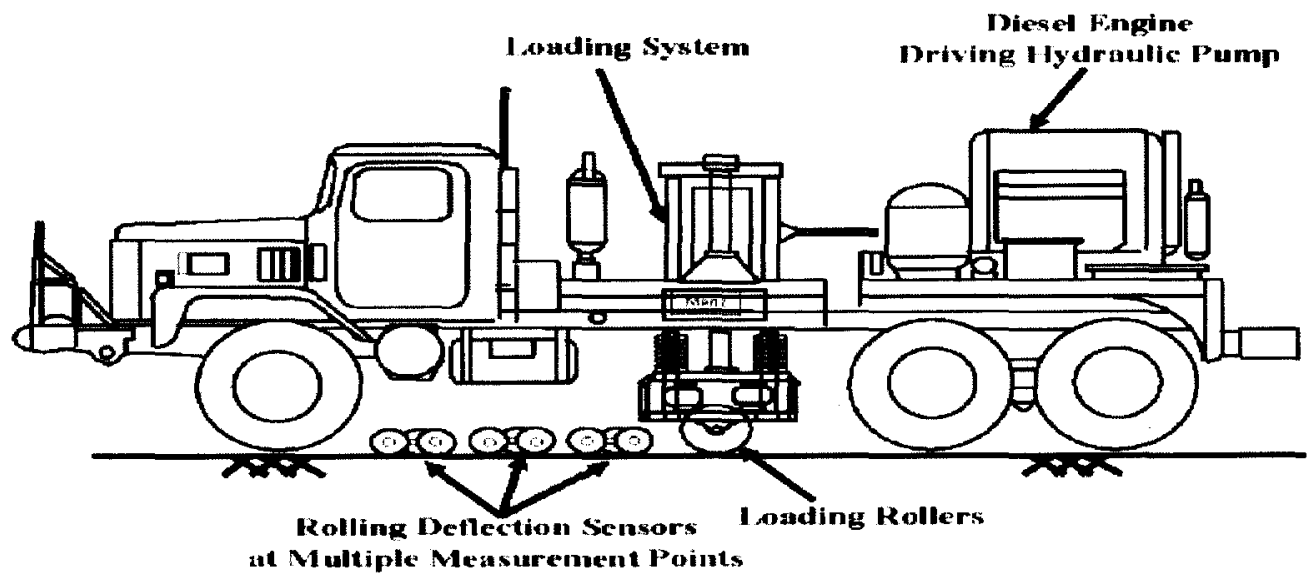

Figure 3: Schematic diagram of a rolling dynamic deflectometer 
- Stationary transient load: This method is commonly known as the Falling weight deflectometer (FWD). Its principle is that a mass with a given weight drops from a certain height on a number of parallel rubber buffers. The buffers act as springs that transfer the impulse load to the pavement surface. The resulting pavement deflections are measured and the captured deflection bowl shape is processed through analyses.

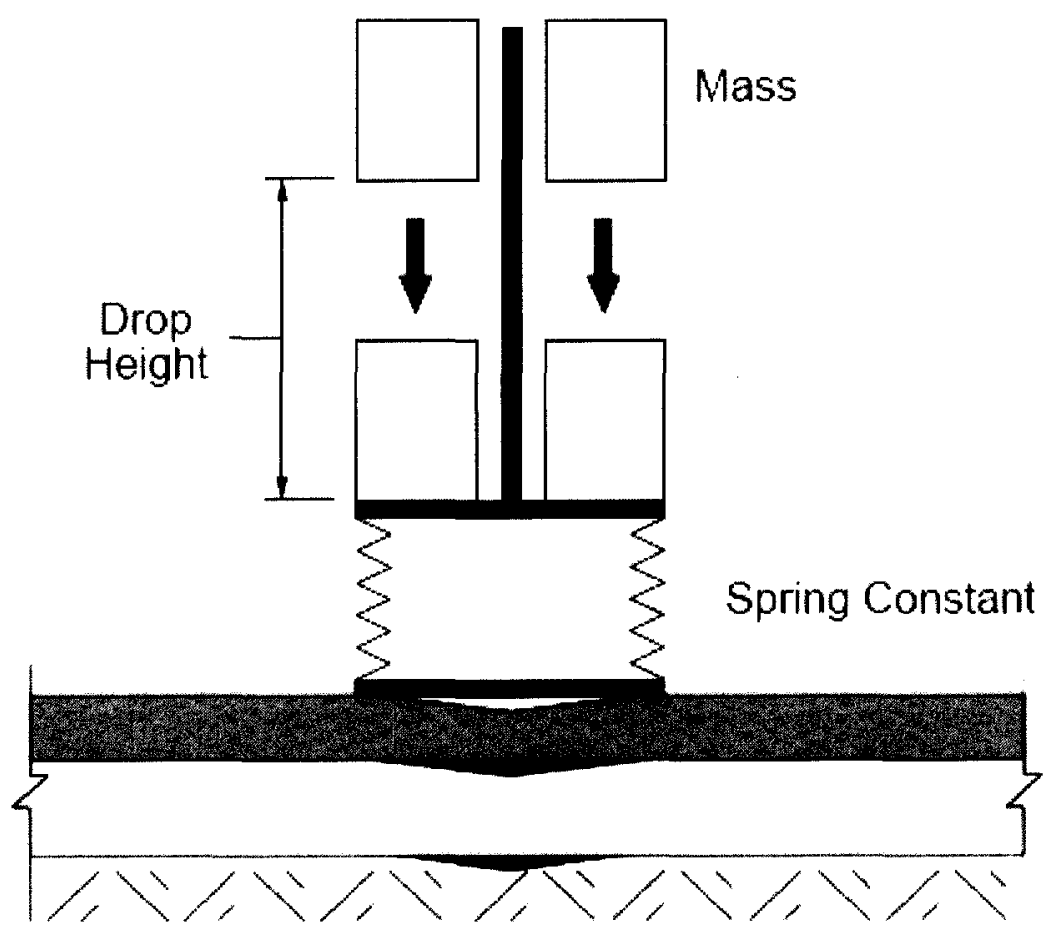

Figure 4: Stationary transient load system 
- Wave propagation: Wave propagation methods for the nondestructive testing of pavements use an impulse force at a point on the pavement surface to generate surface waves. The surface waves are dispersive in layered systems and thus radiate outwards. Two accelerometers acquire the shape of the surface wave as it is transmitted to the pavement. Signal analysis techniques are then applied to the wave form to determine the details of the dispersion that has taken place.

Seismic wave: Seismic methods involve employing a mechanical impulse to generate an acoustic wave that will travel into the ground. The acoustic waves reflect when they encounter subsurface anomalies. The measured reflections, refractions and diffractions of the generated impulse waves are mainly due to the differences of seismic-wave velocity of various subsurface materials.

- Magnetic method: The magnetic method is used to detect small variations in earth's magnetic field. 
Its effectiveness depends on the differences in susceptibility between target bodies with respect to surrounding materials. Magnetic methods are useful in locating ferrous materials, such as oil drums and utility pipes.

For concrete structures, magnetic methods are used in evaluating concrete in a number of ways including: locating and measuring thickness of reinforcements, measure moisture content, corrosion potential, determining pavement thickness, locating defects and corrosion in reinforcements.

- Ultrasonic Pulse Velocity Method: Ultrasonic nondestructive testing uses acoustic energy in the ultrasonic spectrum for evaluating materials. The basic principal is that the pulse velocity of a compressional wave through a medium depends on the elastic properties and density of the medium. The application of the method include: estimation of strength of concrete, homogeneity of concrete and surface crack depth determination among others. It can be performed in two ways: 
- Compression wave (straight beam): this method is used in a cross sectional way, it helps determine thickness measurements, bond testing, plate lamination

- Shear wave (Angle beam): This method is used in a transverse way; it helps determine subsurface defects such as cracks.

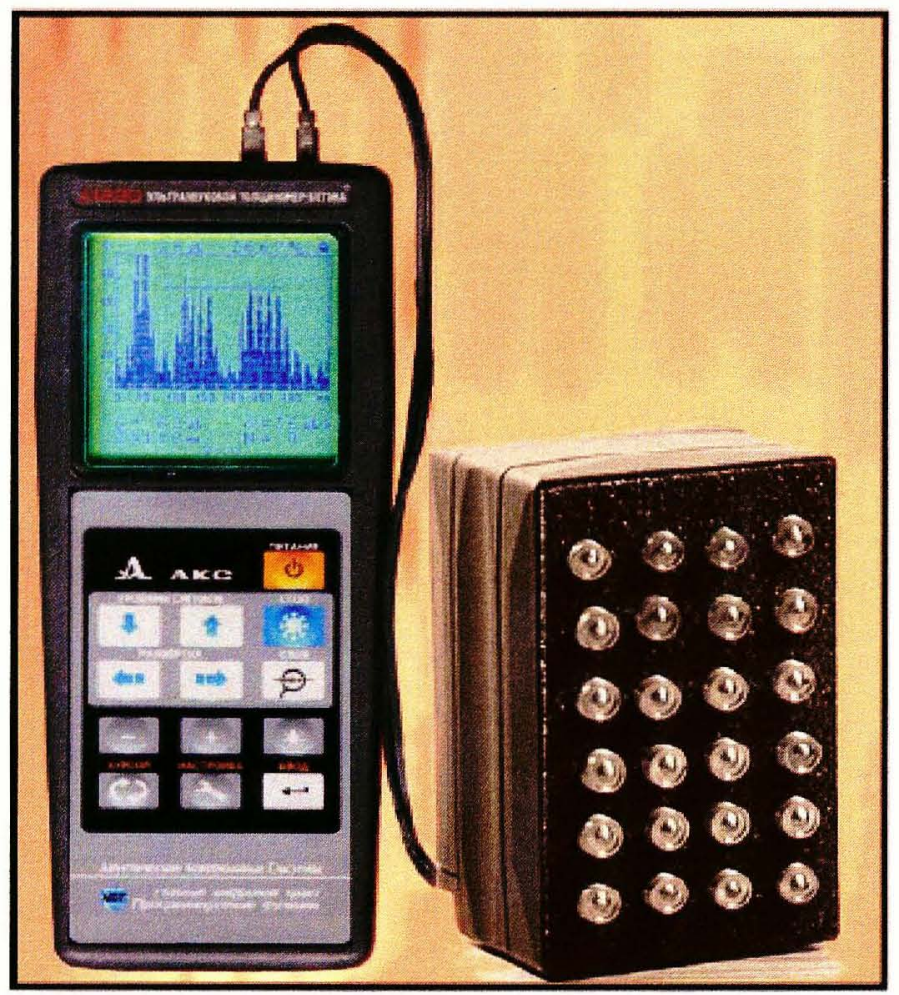

Figure 5: Equipment image Pulse echo system 
- Visual inspection: Visual inspection of pavements by experienced engineers can yield good results in detecting subsurface voids under pavements. The frequency of the inspection is determined by local conditions. "Malvar And Cline recommended that voids may exist where depression are evident after a rainfall or where Concentratic marks are shown after water evaporates; for rigid pavements, the prediction of underlying voids can be made if standard concrete slabs crack into two or more pieces or if faulting occurs at joints" (ZhiYong Zhao, Application of Ground Penetration Radar to Locate Subsurface Voids in Urban Areas, 2005 Page: 29). Cameras and photographs can also be used as part of visual inspection. However this method is limited in that some evidence of the voids must first manifest at the surface. 


\section{CHAP.IV GPR TECHNOLOGY}

GPR is defined as a high resolution, electromagnetic technique designed to investigate shallow subsurface of the earth, building materials, roads and bridges.

The performance of GPR is dependent upon the electrical conductivity of soils. Soils that have high electrical conductivity rapidly attenuate radar energy, restrict penetration depths, and severely limit the effectiveness of GPR. Factors influencing the electrical conductivity of soils include the amount and type of salts in solution and the clay content. (James A Doolittle, Fred E. Minzenmayer, Sharon W. Waltaman and Ellis C. Benham; June 2003: GPR soil suitability maps) 
Ground Penetrating Radar technology is based on electromagnetic theory. A general understanding of electromagnetic waves is beneficial in order to understand GPR capabilities and limitations.

\section{ELECTROMAGNETIC ENERGY}

Electromagnetic energy is a form of energy that is transferred by radiation. It is made of both electric and magnetic energy components and can be viewed as a series of sinusoidal waves of varying wavelength. The resulting electromagnetic waves are uniquely characterized by two parameters: frequency and wavelength.

The alternating electrical current produces an electric and magnetic field that are also alternating: the succession of cycles going "back and forth" constitutes a wave. A wave is a vibration that is propagated in space. 
At high frequencies, the electric and magnetic fields are not separable, and are named electromagnetic waves or electromagnetic fields.

There are many forms of electromagnetic energy including gamma rays, $\mathrm{x}$ rays, ultraviolet radiation, visible light, infrared radiation, microwaves and radio waves.

\section{WAVELENGTH}

The wavelength is the distance between repeating units of a wave pattern. It is commonly designated by the Greek letter $(\lambda)$.

In a sine wave, the wavelength is the distance between the midpoints of the wave. The wavelength is equal to the speed of a wave divided by the frequency of the wave.

\section{FREQUENCY}

The frequency of waves can be defined under two motions : 
- A wave motion: it is the number of waves that pass through a given point per second.

- An oscillation motion: it is the number of cycles per second. These cycles per second are called Hertz (Hz) in honor of nineteenth-century German physicist Heinrich Rudolf Hertz (1857-1894).

\section{PERIOD}

For a transverse wave, a period is the amount of time required to complete one full cycle of the wave, from trough to crest and back to trough. In a longitudinal wave, a period is the interval between waves. With an oscillator, a period is the amount of time it takes to complete one cycle. The value of a period is usually expressed in seconds.

5. ELECTROMAGNETIC SPECTRUM

\begin{tabular}{|l|l|l|l|}
\hline Region & $\begin{array}{l}\text { Wavelength } \\
\text { (Angstroms) }\end{array}$ & $\begin{array}{l}\text { Wavelength } \\
\text { (Centimeters) }\end{array}$ & $\begin{array}{l}\text { Frequency } \\
(\mathrm{Hz})\end{array}$ \\
\hline Gamma Rays & $<0.1$ & $<10^{-9}$ & $>3 * 10^{19}$ \\
\hline $\mathrm{x}-$ Rays & $0.1-10$ & $10^{-9}-10^{-7}$ & $3 * 10^{19}-3 * 10^{17}$ \\
\hline Ultraviolet & $10-4 * 10^{3}$ & $10^{-7}-4 * 10^{-5}$ & $3 * 10^{17}-7.5 * 10^{14}$ \\
\hline
\end{tabular}




\begin{tabular}{|l|l|l|l|}
\hline Visible & $\begin{array}{l}4 * 10^{3}- \\
7 * 10^{3}\end{array}$ & $\begin{array}{l}4 * 10^{-5}-7 * 10^{-} \\
7.5 * 10^{14}- \\
4.3 * 10^{14}\end{array}$ \\
\hline Infrared & $7 * 10^{3}-10^{6}$ & $7 * 10^{-5}-0.01$ & $\begin{array}{l}4.3 * 10^{14}- \\
3 * 10^{12}\end{array}$ \\
\hline Microwave & $10^{6}-10^{9}$ & $0.01-10$ & $3 * 10^{12}-3 * 10^{9}$ \\
\hline Radio & $>10^{9}$ & $>10$ & $<3 * 10^{9}$ \\
\hline
\end{tabular}

Table 1: Electromagnetic spectrum table

Duplicated from Zhiyong Zhao dissertation,

"Application of Ground Penetrating Radar to Locate Subsurface Voids in Urban Areas" 2004, page 10.

\section{RADAR ENERGY}

The word "Radar" is derived from Radio Detection and Ranging and refers to the technique of using radio waves to detect the presence and the distance of a target. Radars operate by transmitting electromagnetic energy into the surrounding environment and detecting energy reflected by objects. If a directive antenna transmits a narrow beam of this energy, the directions from which reflections come and hence the bearing of the object may be estimated. 
The distance to the reflecting object is estimated by measuring the period between the transmission of the radar pulse and reception of the echo. In most radar applications this period will be very short since electromagnetic energy travels with the velocity of light.

Since radio waves travel through air at a known velocity, the distance of the target away from the radar can be determined by measuring the amount of time between the transmission, and the reception of the reflection. Radars were initially designed to detect the presence of aircrafts shortly before and during World War II, but today radars are used in a wide array of applications.

\section{2 PRINCIPLE OF GPR}

Ground Penetrating Radars works much like regular radars. A transmitting antenna emits short pulses of radio frequency electromagnetic energy into the subsurface. The energy passes through the ground and some is reflected back to a receiving antenna. A computer processes the reflected signal, measures the 
strength and time between emission and reception and produces a visual representation of the subsurface. GPR uses pulses of electromagnetic radiation in the microwave band of the radio spectrum.

GPR uses electromagnetic wave propagation and scattering to create images. It images, locates and quantitatively identifies changes in dielectric constants in the subsurface soil. Radar energy is reflected at boundaries of electrically dissimilar materials where there is a contrast in the dielectric constants.

\section{ANTENNA}

The antenna functions as a bridge between the control unit and the target media. It receives a command from the control unit to send an electromagnetic wave in the subsurface. It generates the wave and receives the reflected wave, and it transfers it back to the control unit.

GPR antennas have a wide range of operation frequency; readily available antennas have a range frequencies 
from $100 \mathrm{MHz}$ to $1.5 \mathrm{GHz}$, while antennas of lesser or higher frequency can be made, depending on a survey's requirements. Antenna's of frequencies of $3 \mathrm{GHz}$ and 6 $\mathrm{GHz}$ were developed for concrete testing (Fan-nian Kong, Norwegian Geotechnical Institute, Choice of Antenna Type and Frequency Range for Testing Concrete Structures, 2000), while antennas of frequency of 20 MHz can also have been developed (Chi-Chih Chen and Matthew B. Higgins, OSU. A New Ultra-wide Bandwidth Horn-fed Dipole GRR Antenna Design, 2000)

GPR antennas usually fall under two broad categories:

- Ground coupled antennas: Ground coupled antennas sit directly on the surface of the ground being analyzed and they are suitable for deep penetration.

- Air coupled antennas: Air coupled antennas operate without touching he ground surface. They can be mounted to vehicles, airplanes or satellites.

GPR antennas can also be classified as mono-static or bi-static. Mono-static antennas have the transmitter and receiver integrated in one single unit, whereas 
Bi-static antennas use two separate units, one unit acting as a transmitter and the other as a receiver.

The antenna choice is very critical for data acquisition, as it needs to be matched to the site's conditions and the experiments objectives.

The choice of the antenna for a specific location will depend among other needs on the objective of the survey, the size and type of work required, the depth of survey required and the terrain accessibility. Usually the most important factor to consider is the depth required as this will influence the choice of the frequency to use. As frequency increases the depth of penetration reduces.

\section{ELECTRICAL CONDUCTIVITY}

GPR energy is subjected to attenuation as it moves through a material as it is a electromagnetic energy. GPR signals are able to penetrate deeper in the material if the material is resistant (low conductivity). If the material is conductive GPR energy gets absorbed very quickly; this is the case 
for salty water or in general for high moisture content materials.

\section{DIELECTRIC CONSTANT}

The dielectric constant is a measure of the extent to which a substance concentrates the electrostatic lines of electric flux. It is the ratio of the amount of electrical energy stored in a substance, when a static electric field is imposed across it, relative to vacuum (which has a dielectric constant of 1). The dielectric constant is also known as the static permittivity.

In general, the dielectric constant is a complex constant, with the real part giving reflective surface properties (Fresnel reflection coefficients), and the imaginary part giving the radio absorption coefficient

Dielectric constant is an important factor that influences the speed and the propagation of the Electromagnetic wave in a subsurface media. Higher dielectric values mean slower travel time and shallower penetration. 


\section{MATERIAL DIELECTRIC CONSTANT DETERMINATION}

In order to survey and process data using GPR, the dielectric value of the surveyed media needs to be known. Methods used to determine the dielectric value include amplitude method, velocity method, instrument method or use of available data from various agencies. (zHAO dissertation), the use of combined embedded modulated scattering techniques and near-field microwave NDT method was found to be effective (Dana Hughes and Reza Zoughi, senior member, IEEE; Dec 2005) .

A dielectric material is a substance that is a poor conductor of electricity, but an efficient supporter of electrostatic field.

\section{DETECTION METHODS}

GPR antenna signals are propagated not only straight into the subsurface but also to the sides. As a result, GPR detects responses from features which are not directly below them but also at a small distance. This characteristic gives rise to the classic 
hyperbolic signature of localized features such as pipes, cables or other spatially limited objects. 


\section{CHAP.V EQUIPMENT}

This chapter illustrates the components of the GPR equipment used during the experiments. The equipment used is part of the GSSI TerraSIRch SIR System-3000 manufactured by GSSI (Geophysical Survey Systems, Inc). Materials used for most of this chapter are referenced from GSSI publications. These publications are TerraSIRch SIR System-3000 User's manual and the RADAN 6.5 User's manual.

\section{V.1 COMPONENTS OF THE EQUIPEMENT}

The SIR-3000 is composed of the following items:

\section{CONTROL UNIT}

A digital control unit (DC-3000) with preloaded operating system: the major parts of the control unit 
are the keypad, color SVGA video screen, connector panel, battery slot, indicator lights. The video screen allows viewing the data in real time or playback mode.

The control unit stores the data and could be used to analyze them. The control unit is the source of the electromagnetic wave and it stores the returned waves' reflections from the subsurface media.

\section{POWER SUPPLY}

The power source of the system can originate from batteries as well as direct electrical connections through an $\mathrm{AC}$ adapter.

\section{ANTENNA}

The TerraSIRch SIR System offers a range of different antennas that are chosen based on the type of work required. The antenna used during the experiments has a frequency of $1.5 \mathrm{GHz}$. 


\section{SURVEY WHEEL}

The main objective of a survey wheel is to measure horizontal distances surveyed. It is usually attached to large wheel carts but could also be used as part of a wheel cart. In the current case, the wheel cart for the $1.5 \mathrm{GHz}$ antenna also acts as the survey wheel.

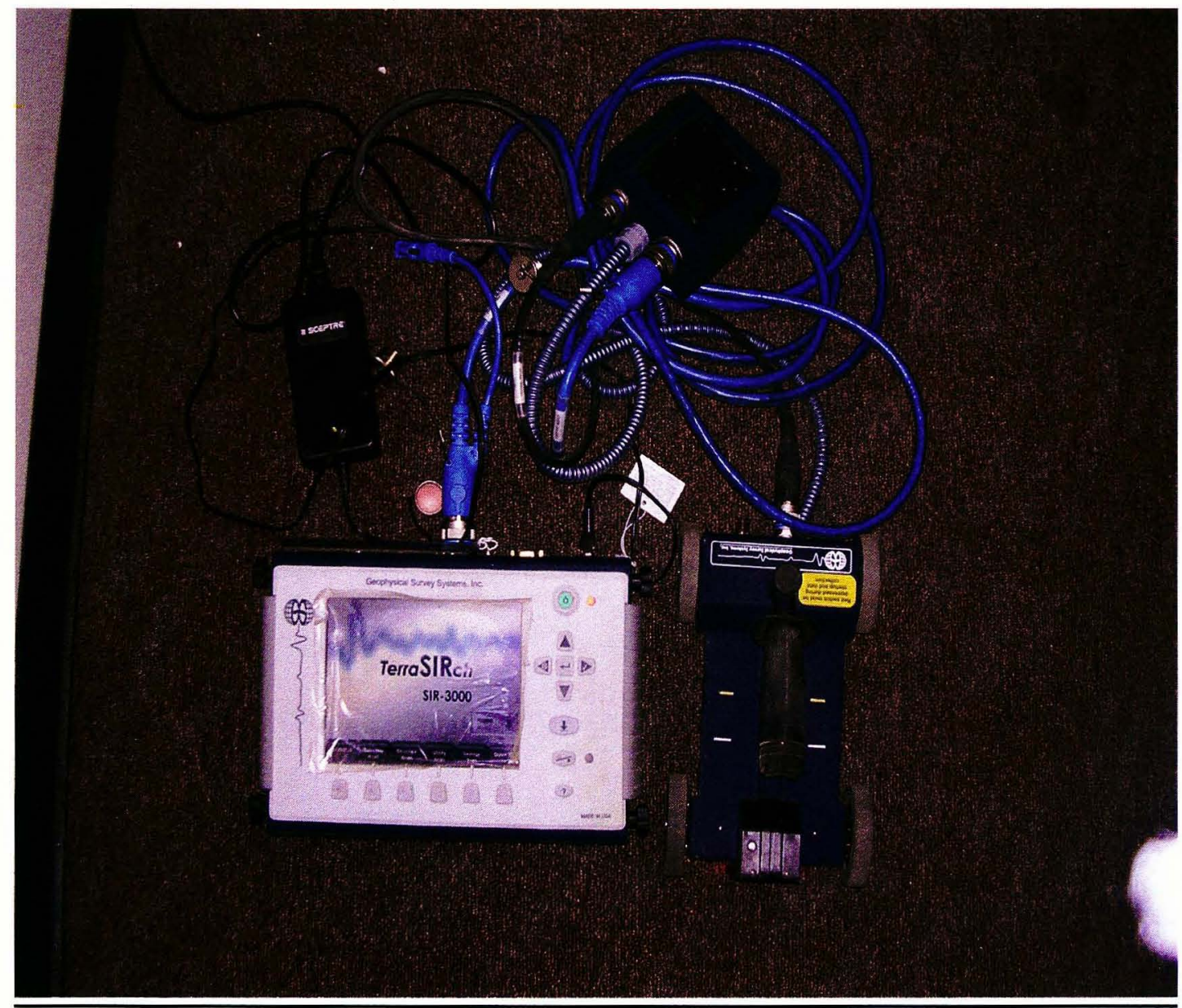

Figure 6: Image of the equipment 


\section{V.2 EQUIPMENT OPERATION}

\section{HARDWARE SETUP}

Before the system is used, hardware components need to be connected in the following order:

1. The antenna has to be attached to the minicart. It has to be positioned all the way forward in the cart.

2. The antenna is connected to the system by a control cable and the cable is plugged back into the SIR3000 .

3. Before plugging the SIR-3000 to either external power source or inserting a battery, all connections are checked to ensure that they are properly connected to the survey wheel and to the electronic box.

2. DATA ACQUISITION

Data acquisition is undertaken after hardware setup is finished. When the SIR-3000 is started, the system 
boots up and an introductory screen is shown with the words TerrasIRch, SIR-3000.

Three acquisition modes are normally used in the field:

- Continuous acquisition mode: The method is used to conduct a quick survey with no need to precisely measure the survey distance. This is mostly used in geophysical and environmental evaluations.

- Survey wheel acquisition mode: The method is used when precise distance measurements are needed. A survey wheel is connected to the antenna through a cable in order to precisely measure the surveyed distance. Horizontal survey markers are used equivalent to the horizontal distance traveled.

- Point acquisition mode: Also known as impulse acquisition mode, it is used on rough terrain when continuous data can not be acquired, or signal strength is too weak to penetrate to necessary depth. It is also used when maximum signal enhancement is required. The system collects data 
only when the external marker is pressed. It has been shown that this mode has a wide range of applications and can be used for regular inspection and for identifying the cause of damage of concrete structures such as concrete bridges, anchors and dowels in concrete highways successfully (C. Maierhofer, T. Kind: Federal Institute for Materials Research and Testing Berlin Germany, Application of Impulse Radar for NDT investigation of Concrete Structures, 2002):

Collection modes are selected depending on the project being surveyed; a choice of 6 different collection modes are offered by the TerrasIRch, SIR-3000, Four of them are pre-set and are limited in their customization as they are meant for specific applications or to collect data for on-screen target location as opposed to post processing. The other modes are fully customizable.

The preset modes are:

- ConcreteScan: ConcreteScan is meant for a quick scan of concrete, it is used to locate shallow structural 
features in concrete and marking their locations directly on the survey surface before any cutting, coring or drilling takes place. It works with the 1.5 GHz and $900 \mathrm{GHz}$ antennas.

- structurescan: Structurescan is used to collect very high resolution $3 \mathrm{D}$ data over a concrete slab or wall with the $1.5 \mathrm{GHz}$ antenna and GSSI's scan pad. The survey produces a 3D cube of data that can be viewed in plain view at differing paths so as to note clear locations for coring and cutting.

- UtilityScan: UtilityScan is meant for a quick examination of an area in order to locate utilities in soils under concrete slabs or asphalt. Utilityscan can be used with $200 \mathrm{MHz}, 400 \mathrm{MHz}$ and $900 \mathrm{MHz}$ antennas. Utilityscan allows the creation of 2D profiles in order to mark the location of utilities prior to trenching or excavations.

- Geologyscan: GeologyScan mode is optimized for 2D profiling of geological features such as high bedrock, sediments bedding, water tables... It is also 
useful for archeological profiling. It works with $400 \mathrm{MHz}, 200 \mathrm{MHz}$ and $100 \mathrm{MHz}$ antennas.

\section{PARAMETER SETUP}

Selection and setup of operating parameters is vital for the success of a GPR survey project; it is an important step towards a successful data acquisition. This section will provide a description of some of the parameters that need to be set before surveys are carried out.

Parameters setup is done under the collect menu of the TerraSIRch, SIR-3000. These parameters are:

- Survey units: Selection of survey units for depth and distance has to be done, choosing either the Metric system or the English system. An appropriate scale can be chosen; the scale determines whether to display distance in time or feet.

- Antenna selection: The radar / antenna selected for the survey is entered along with its transmission rate and mode. The transmission rate defines how 
fast an antenna will collect the data whereas the mode selection allows selection of either the point data collection mode, the distance data collection mode or the time based data selection mode.

- Samples / scan: This parameter sets the number of data samples in a vertical scan. The more samples collected the better the vertical resolution will be. Choice is made from a list of 256, 512 1024, 2048, 4096 or 8192 samples per scan. As the sample numbers increase, maximum scan rate drops and the survey file becomes larger. It is recommended to use 512 or 1024 samples / scan for most applications.

- Range: The time range is a parameter that functions with the target depth and the medium's properties. It is proportional to the depth viewed. A longer time range displays energy reflected from deeper distances. Range is a two way travel time.

\begin{tabular}{|l|l|l|l|}
\hline Frequency & Sample application & $\begin{array}{l}\text { Typical max } \\
\text { depth Feet } \\
\text { (meter) }\end{array}$ & $\begin{array}{l}\text { Typical } \\
\text { range } \\
\text { (ns) }\end{array}$ \\
\hline $1.5 \mathrm{GHz}$ & $\begin{array}{l}\text { Structural concrete, } \\
\text { Roadways, Bridge } \\
\text { decks. }\end{array}$ & $1.5(0.5)$ & $10-15$ \\
\hline $900 \mathrm{MHz}$ & Concrete, Shallow & $3(1)$ & $10-20$ \\
\hline
\end{tabular}




\begin{tabular}{|c|c|c|c|}
\hline & soils, Archaeology. & & \\
\hline $400 \mathrm{MHz}$ & $\begin{array}{l}\text { Shallow geology, } \\
\text { Utility, } \\
\text { Environment, } \\
\text { archaeology }\end{array}$ & $12(4)$ & $20-100$ \\
\hline $200 \mathrm{MHz}$ & $\begin{array}{l}\text { Geology, } \\
\text { Environmental }\end{array}$ & $25(8)$ & $70-300$ \\
\hline $100 \mathrm{MHz}$ & $\begin{array}{l}\text { Geology, } \\
\text { Environmental }\end{array}$ & $60(20)$ & $300-500$ \\
\hline
\end{tabular}

Table 3: Typical range values for different GPR antenna frequencies (Duplicated from "TerrasIRch SIR3000 User's Manual" 2004, page 61.)

- Dielectric constant: This parameter reflects the velocity that radar energy moves through the material. The table below provides some known values for typical material dielectric constants.

\begin{tabular}{|l|l|l|}
\hline Material & $\begin{array}{l}\text { Dielectric } \\
\text { constant }\end{array}$ & $\begin{array}{l}\text { Velocity } \\
\text { (mm/sec) }\end{array}$ \\
\hline Air & 1 & 300 \\
\hline Water (fresh) & 81 & 33 \\
\hline Water (sea) & 81 & 33 \\
\hline Polar snow & $1.4-3$ & $194-252$ \\
\hline Polar ice & $3-3.15$ & 168 \\
\hline Temperate ice & 3.2 & 167 \\
\hline Pure ice & 3.2 & 167 \\
\hline $\begin{array}{l}\text { Freshwater lake } \\
\text { ice }\end{array}$ & 4 & 150 \\
\hline Sea ice & 2.5 & $78-157$ \\
\hline Permafrost & $1-8$ & $106-300$ \\
\hline $\begin{array}{l}\text { Coastal sand } \\
\text { (dry) }\end{array}$ & 10 & 95 \\
\hline Sand (dry) & $3-6$ & $120-170$ \\
\hline Sand (wet) & $25-60$ & $55-60$ \\
\hline
\end{tabular}




\begin{tabular}{|l|l|l|}
\hline Silt (wet) & 10 & 95 \\
\hline Clay (wet) & $8-15$ & $86-110$ \\
\hline Clay soil (dry) & 3 & 173 \\
\hline Marsh & 12 & 86 \\
\hline $\begin{array}{l}\text { Agricultural } \\
\text { land }\end{array}$ & 15 & 77 \\
\hline Pastoral land & 13 & 83 \\
\hline Average soil & 16 & 75 \\
\hline Granite & $5-8$ & $106-120$ \\
\hline Limestone & $7-9$ & $100-113$ \\
\hline Dolomite & $6.8-8$ & $106-115$ \\
\hline Basalt (wet) & 8 & 106 \\
\hline Shale (wet) & 7 & 113 \\
\hline Sandstone (wet) & 6 & 112 \\
\hline Coal & $4-5$ & $134-150$ \\
\hline Quartz & 4.3 & 145 \\
\hline Concrete & $5-8$ & $55-120$ \\
\hline Asphalt & $3-5$ & $134-173$ \\
\hline PVC & 3 & 173 \\
\hline
\end{tabular}

Table 4: Dielectric constant values of some common materials (Duplicated from "TerraSIRch SIR-3000 User's Manual" 2004, page 71.)

\section{- Rate:}

- Scan / second: This is the number of scans that the system records per second. It controls the horizontal sample rate when the system is set to the continuous mode.

- Scans / unit: Selection of scans per unit horizontal length. This is the scan spacing when the survey wheel is used to collect data. 
- Gain: Gain is the artificial addition of power to the signal in order to counteract the natural effects of attenuation. It amplifies the amplitude of weak signals. It should be adjusted to correspond to different soil conditions. It is difficult to identify data for which the amplitude is small if the gain is set too low, but a high gain might loose data of high amplitude signal.

- Position: This parameter controls the position of time zero. Time zero is the location of the beginning of the scan. Also an offset position and surface position have to be set.

- Filters: It allows the removal of interference or smooth noise. This parameter is used to either eliminate high frequency signal beyond a specified value or a low frequency lower than a specified value. 
Radar can be viewed under five different formats: LineScan format, Wiggle format, O-Scope format, LineScan with wiggle format and 3D display.

The images for the different data display formats represent a steel rebar $3^{\prime \prime}$ deep in the sand box.

- Linescan format: The linescan format is the most useful in mapping underground man-made objects. Data is displayed in a color amplitude form. Specific positive or negative amplitudes of recorded signals are represented by certain colors depending upon the color table and color transforms selected.

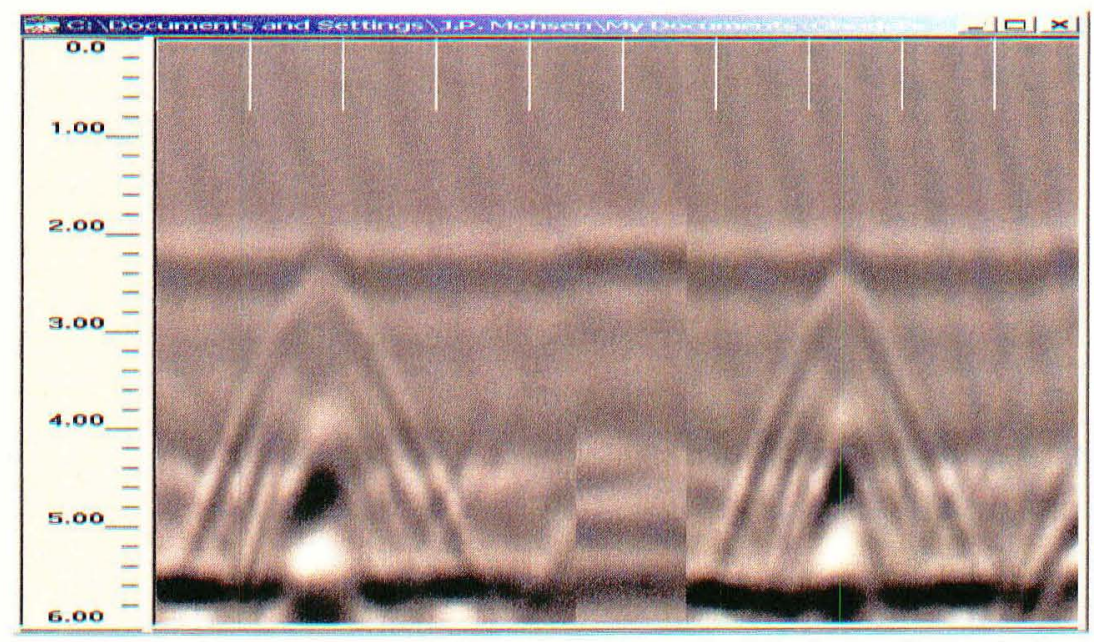

Figure 7: LineScan format sample 
- Wiggle format: The wiggle format is useful in identifying geological features. It consists of multiple radar scans displayed in a waveform (wiggle traces). The data file is displayed with the beginning of each scan on top and time (or depth) increasing downward.

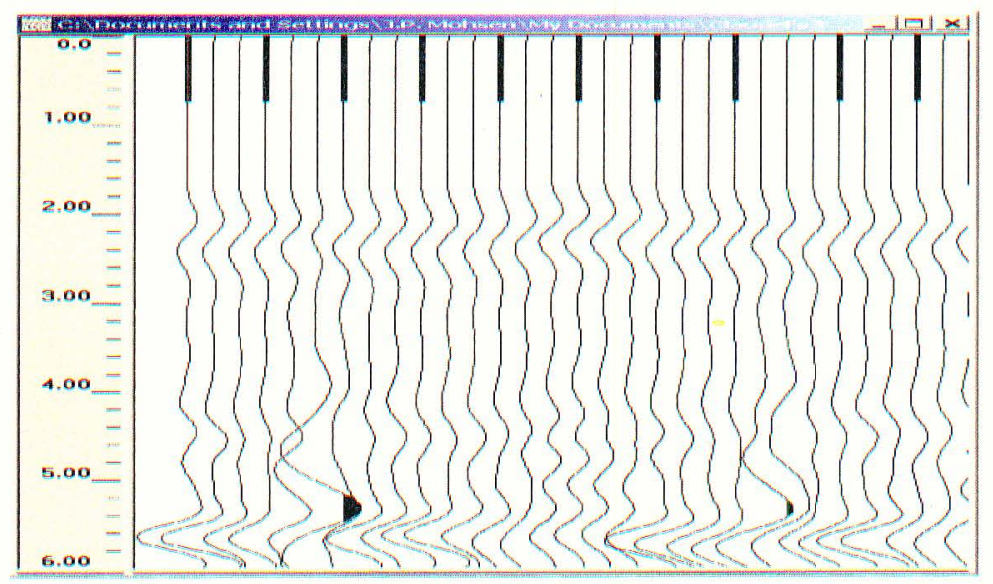

Figure 8: Wiggle format sample

- o-scope format: The O-scope format is used to view a single waveform or wiggle trace. Data is viewed one scan at time.

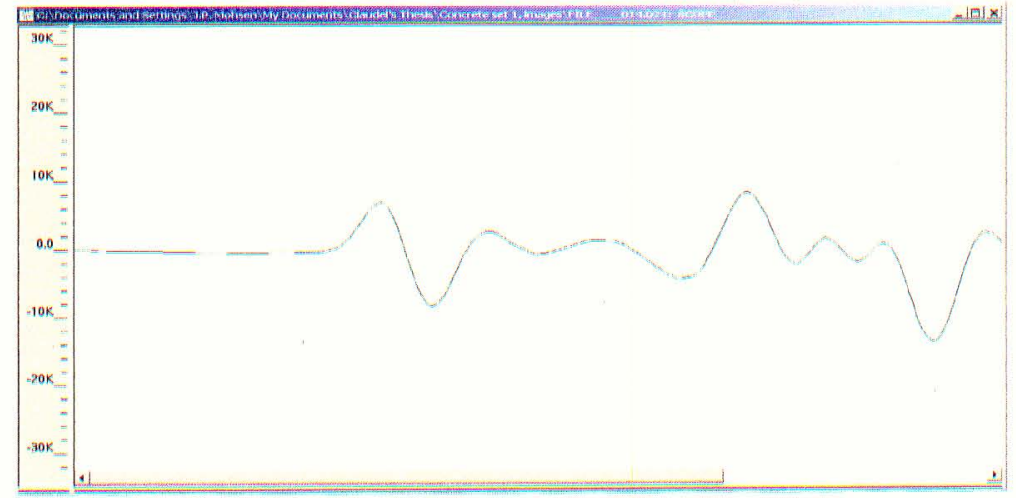

Figure 9: O-scope format sample 
- Linescan with wiggle format: This is a combination of the linescan format and wiggle format. Data is viewed as in linescan format with a wiggle trace window that scrolls automatically when the display cursor is placed in the linescan window and the left mouse button is clicked on the display.

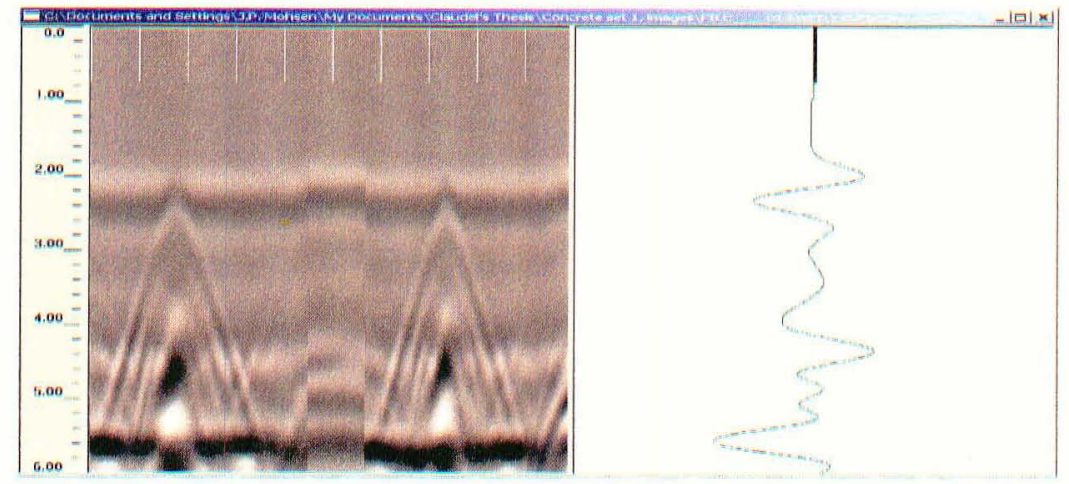

Figure 10: Line scan with wiggle format sample

- 3 D display format: The 3 D display format is used to view 3 D files or a single profile in $3 \mathrm{D}$. and use the 3 D display tools to analyze it.

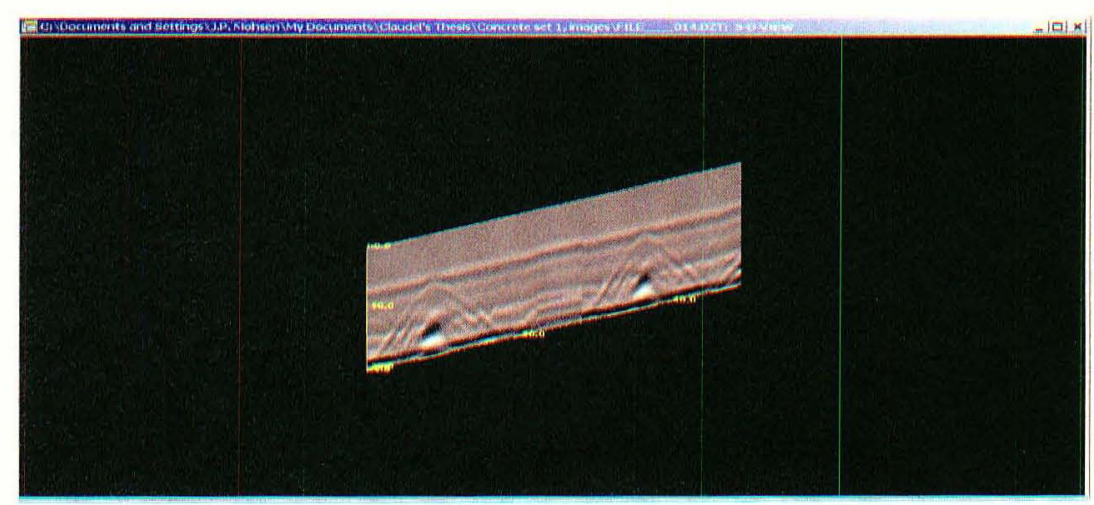

Figure 11: 3D display format sample 


\section{DATA PROCESSING}

Most of $2 \mathrm{D}$ data can be viewed and analyzed as raw data. But, at certain times it is necessary to apply some processing procedures in order to have better GPR signals to study. Processing is done to achieve the following:

- To remove unwanted signal (noise) from the data and thereby improve data interpretation.

- To correct for geometric errors and provide more accurate spatial and depth interpretation.

- To convert from time to depth and provide accurate information in depth sections.

- To provide displays that are easier to understand than the raw data.

Raw GPR data can be edited. Functions to remove unwanted parts of the data, to zoom an image, or to 
add markers changing the color table to the raw data are available.

The following are some of the processing functions for further processing available after the initial editing:

- Horizontal and vertical filters.

- Spatial 2 D filtering.

- Range gain.

- Restore gain.

- Surface Position Adjustment.

- Distance Normalization.

- Horizontal scaling.

- Horizontal Distance adjustment.

- Surface Normalization.

- Velocity analysis.

Process objectives and method to accomplish them:

\begin{tabular}{|l|l|}
\hline Objective & Methods \\
\hline $\begin{array}{l}\text { Remove flat-lying } \\
\text { ringing system noise }\end{array}$ & Horizontal High Pass \\
\end{tabular}




\begin{tabular}{|c|c|}
\hline & $\begin{array}{l}\text { - Vertical High Pass Filter. } \\
\text { - Spatial Filter. } \\
\text { - Background Removal. }\end{array}$ \\
\hline High frequency noise & $\begin{array}{l}\text { - Vertical Low Pass Filter. } \\
\text { - Horizontal Low Pass } \\
\text { Filter. } \\
\text { - Spatial Filter. }\end{array}$ \\
\hline Ringing multiple & - Deconvolution. \\
\hline $\begin{array}{l}\text { Remove diffraction and } \\
\text { correct dipping layers. }\end{array}$ & - Migration \\
\hline $\begin{array}{l}\text { Increasing visibility } \\
\text { of low amplitude } \\
\text { features }\end{array}$ & $\begin{array}{l}\text { - Arithmetic Functions. } \\
\text { - Display and Range Gain. }\end{array}$ \\
\hline Detect subtle features & $\begin{array}{l}\text { - Hilbert Magnitude } \\
\text { Transform. } \\
\text { - Spatial Filter. }\end{array}$ \\
\hline $\begin{array}{l}\text { Generate clearer data } \\
\text { display for reports }\end{array}$ & $\begin{array}{l}\text { - Arithmetic Functions. } \\
\text { - Local Peaks. } \\
\text { - Static Corrections. }\end{array}$ \\
\hline
\end{tabular}

Table 5: Processing objectives and methods 


\section{CHAP . VI METHODOLOGY}

A library of GPR images is developed using known targets set in a sand box.

In this experiment sand was used as the survey medium. Sand allows temporary setups as in sand it is easy to place and replace fixtures such as pipes and steel rebars, also configurations can be changed faster than in a concrete structure.

The Dielectric range of dry sand is fairly close to that of Portland cement concrete, thus GPR images obtained by using sand can be used to simulate GPR images in a concrete slab or structure.

The experimentation used steel rebars as the experiments features. Approximately 500 different images were taken for this experiment. 
The developed images can be categorized under two broad categories:

- Images of the same configuration taken with different setup parameters in order to study the effect of those parameters on the resulting data images.

- Images of different configurations taken with similar parameter setups with an objective of finding the GPR equipment's capacity.

\section{VI.1 EXPERIMENT PROCEDURES}

The steps followed during the experiments are:

1 Configure materials within the sand box.

2 Collect data pertaining to the set-up configuration with initial set parameters.

3 Change parameters and collect data again.

4 Change material configuration and repeat the above steps. 


\section{2 PARAMETERS USED}

The experiment was done under the Concretescan preset mode.

1. PARAMETERS THAT CAN BE CUSTOMIZED

- Antenna (1.5 GHz or $900 \mathrm{MHz})$,

- Select Distance or Time collection mode,

- Maximum penetration depth from a number of presets: $6,12,18,36$ inches.

- SCN/UNIT from 5/7.5/10 per inch.

- Concrete type from a number of presets ranging from Dry to wet.

2. PARAMETERS THAT ARE PRESET

- T_RATE : 100 .

- Sample / scan: 512 deep, 256 shallow.

- Format / Bits: 16.

- RATE : 100 (deep) 200 (shallow)

- Gain: 5 points Auto. 
- Filters.

- Offset position.

- Surface position.

\section{VI.3 SAND BOX}

The sand box is a square of $32 * 32 * 12$ inches in size.

The sand used is obtained from the Ohio River.

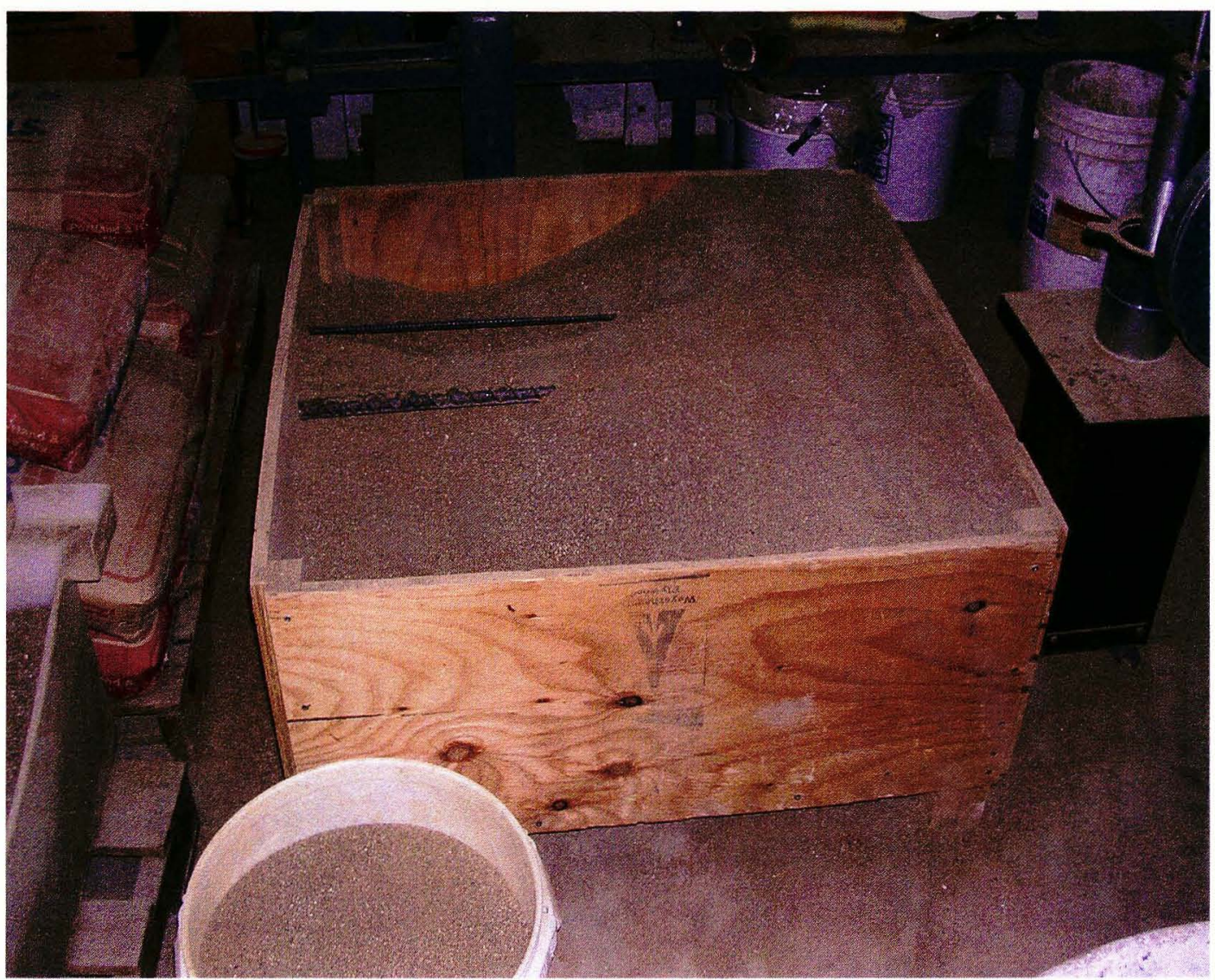

Figure 12: Sand box 


\section{4 MATERIAL USED}

Different sizes of steel rebars were used during the experiments. The sizes of the steel rebars vary from No 3 steel rebars to No9.

\section{VI.5 DATA COLLECTION}

The proper sand dielectric value to use was determined by taking different images of a buried steel pipe at different dielectric values. The proper dielectric value was found by selecting the dielectric value that showed the feature at its exact depth.

Collection of data consisted of first setting up the configuration of the features and then take different GPR images by changing survey setup operating parameters. Each image obtained was recorded for future reference. 


\section{CHAP.VII DATA ANALYSIS \& DISCUSSION}

The analysis is done on raw data without any processing except an increase of the horizontal scale as necessary. GPR images can be analyzed directly or through processing steps that depend on the results desired.

All images obtained during the survey will not be used in the analysis but will be catalogued in the GPR library. The recorded and presented data will be in the following formats:

\begin{tabular}{|l|l|}
\hline Data format & Data presentation \\
\hline Display Format & Linescan \\
\hline Color Table & Grayscale \\
\hline Measurement units & Inches \\
\hline Coordinate lines & Distance traveled \\
\hline
\end{tabular}

Table 6: Data display mode. 
GPR images obtained for metallic cylindrical objects produce parabolic shapes. The pattern of the parabolas is shown below. In the grayscale color table display, the parabola has a prominent white band as shown in figure 13.

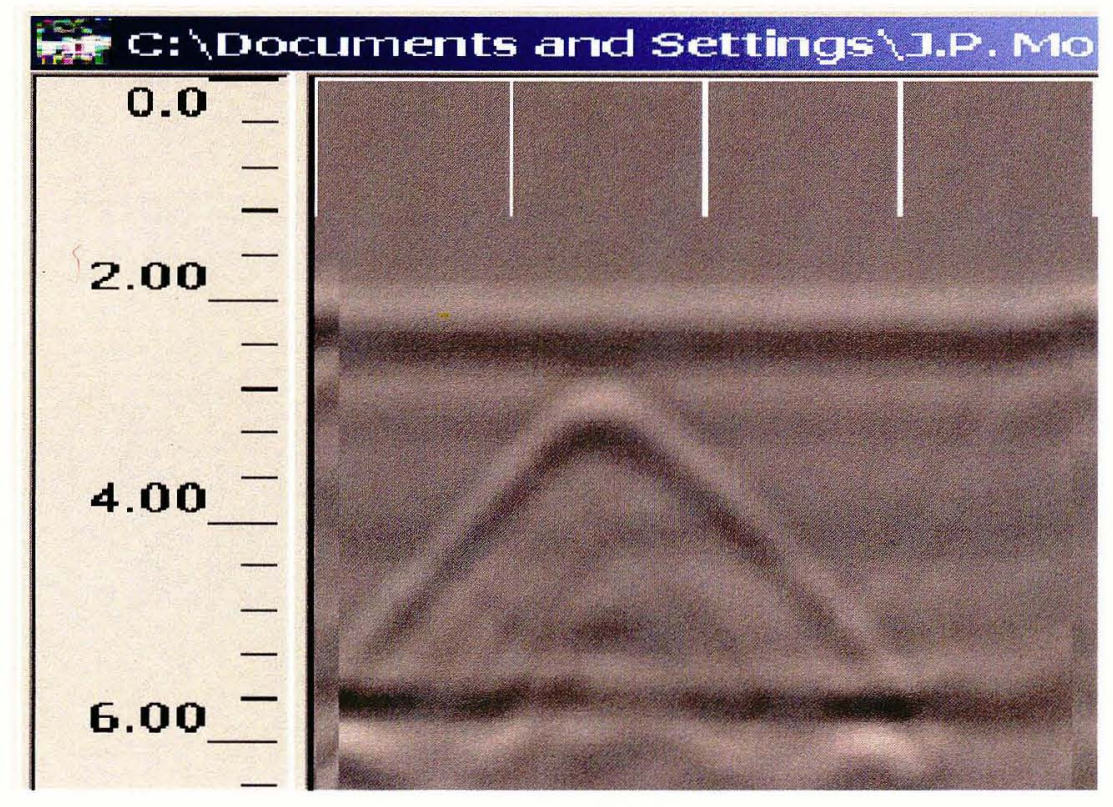

Figure 13: A steel rebar GPR signal

\section{VII.1 SCAN SPACING EFFECTS}

The unit for this parameter is scan per inch. Under the scan per unit parameter, three choices are offered; they are 5 scans per inch, 7.5 scans per inch and 10 scans per inch. 
The effect of the scan per unit parameter is that it determines how detailed the survey results images will be. An increase in the scan spacing number increases the details of the survey image but requires a larger storage volume.

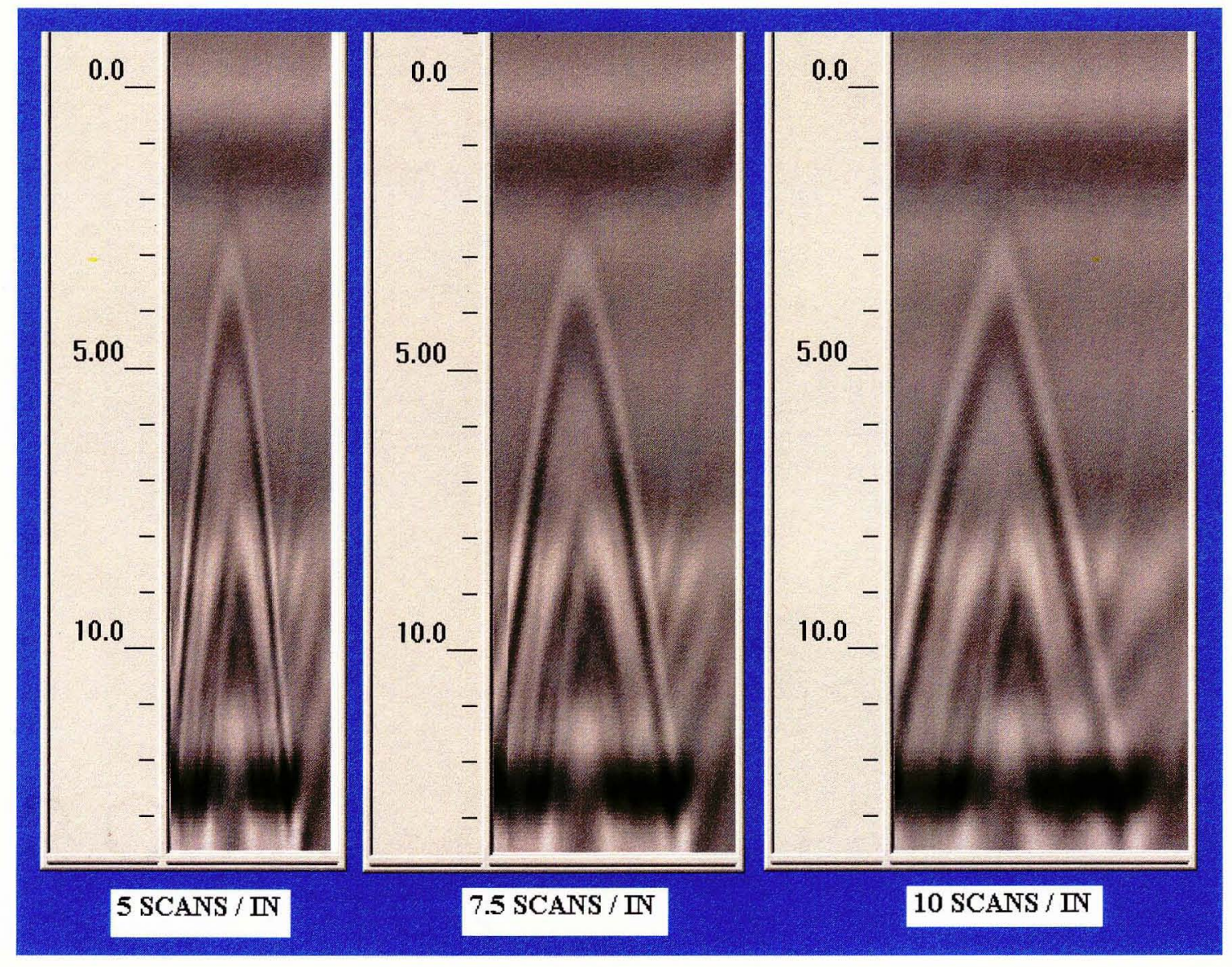

Figure 14: Scan spacing effects of a No 9 steel rebar, 3" deep 
Figure 14, shows a No 9 steel rebar 3" deep. The figure shows the same configuration surveyed with different scan spacing settings. The first was at 5 scans per inch, the second at 7.5 scans per inch and the third at 10 scans per inch. It can be noticed that in general the images look similar but that they are scaled to different sizes; as the scan per unit value increases the images get larger and the parabolas have a wider base.

VII. 2

\section{DEPTH PARAMETER EFFECTS}

The depth parameter is set depending on the depth of the structure surveyed and the depth intended to be shown for the GPR signals images. In Concretescan mode, depth can be set at 6", 12", 18", 36" and 72".

For the purpose of this study, the depth parameter was only varied between 6", 12" and 18". This is due to the size of the sand box.

Figure 15 shows three images of one configuration taken by varying the depth of survey only. The configuration is of a No 9 steel rebar $3^{\prime \prime}$ deep. The 
scan spacing was set at 5 Scans / inch in all cases. Increasing the depth of the shown signals affects the result images by increasing the shown depth, thus images with higher depth will be less detailed. Images with lower depths will be more detailed than those with deeper depths, but the decision of what appropriate depth parameter to select depends on the survey's requirements.

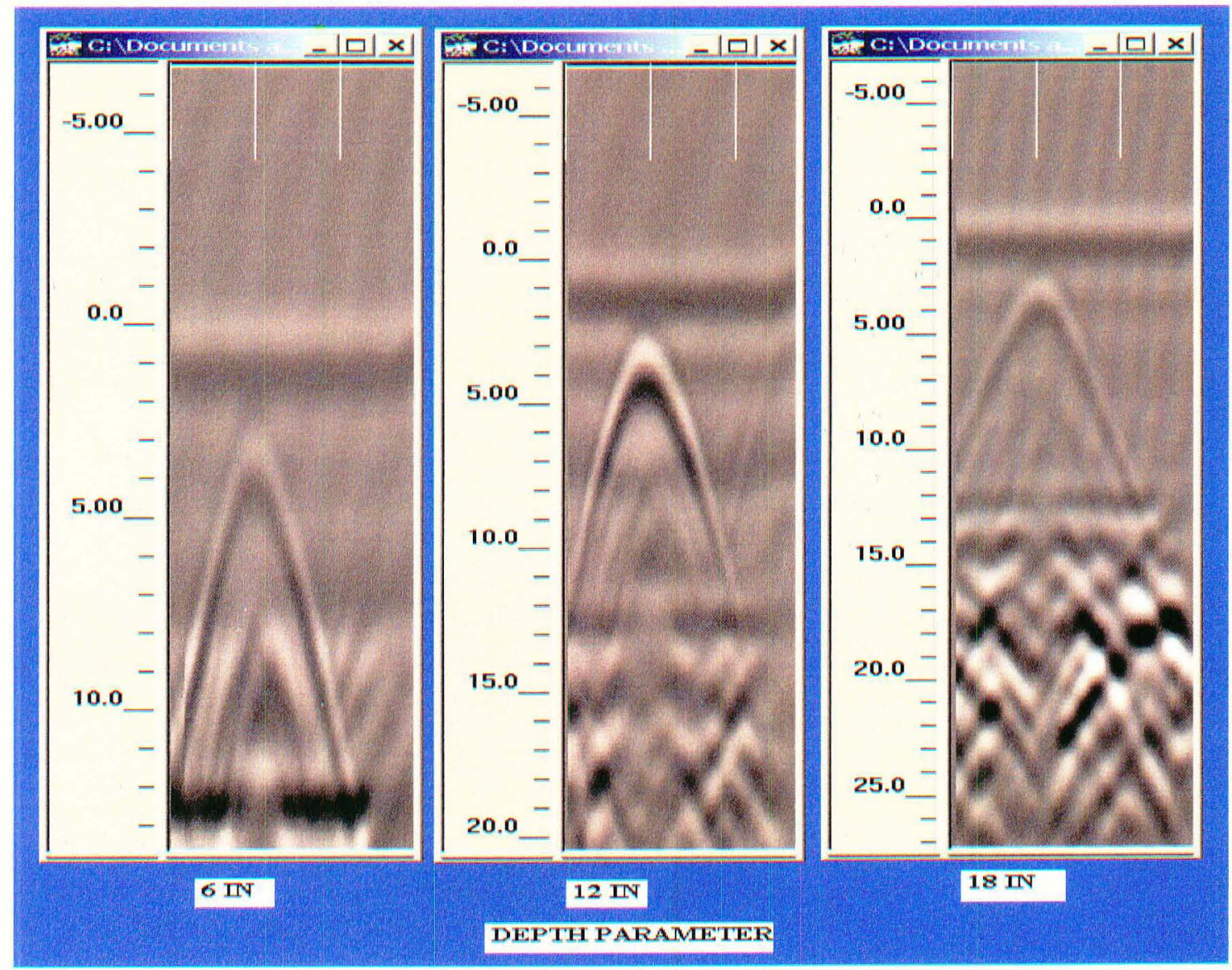

Figure 15: Depth parameter effects of a No 9 steel rebar, 3" deep 
Fig 16 shows different configurations of a No9 steel rebar placed at different depths. The steel rebar is buried 1" deep, 2" deep, 3" deep and 5" deep. The images obtained do not reflect any change in the clarity of the parabola but they clearly show that the steel rebars are not at the same depth.

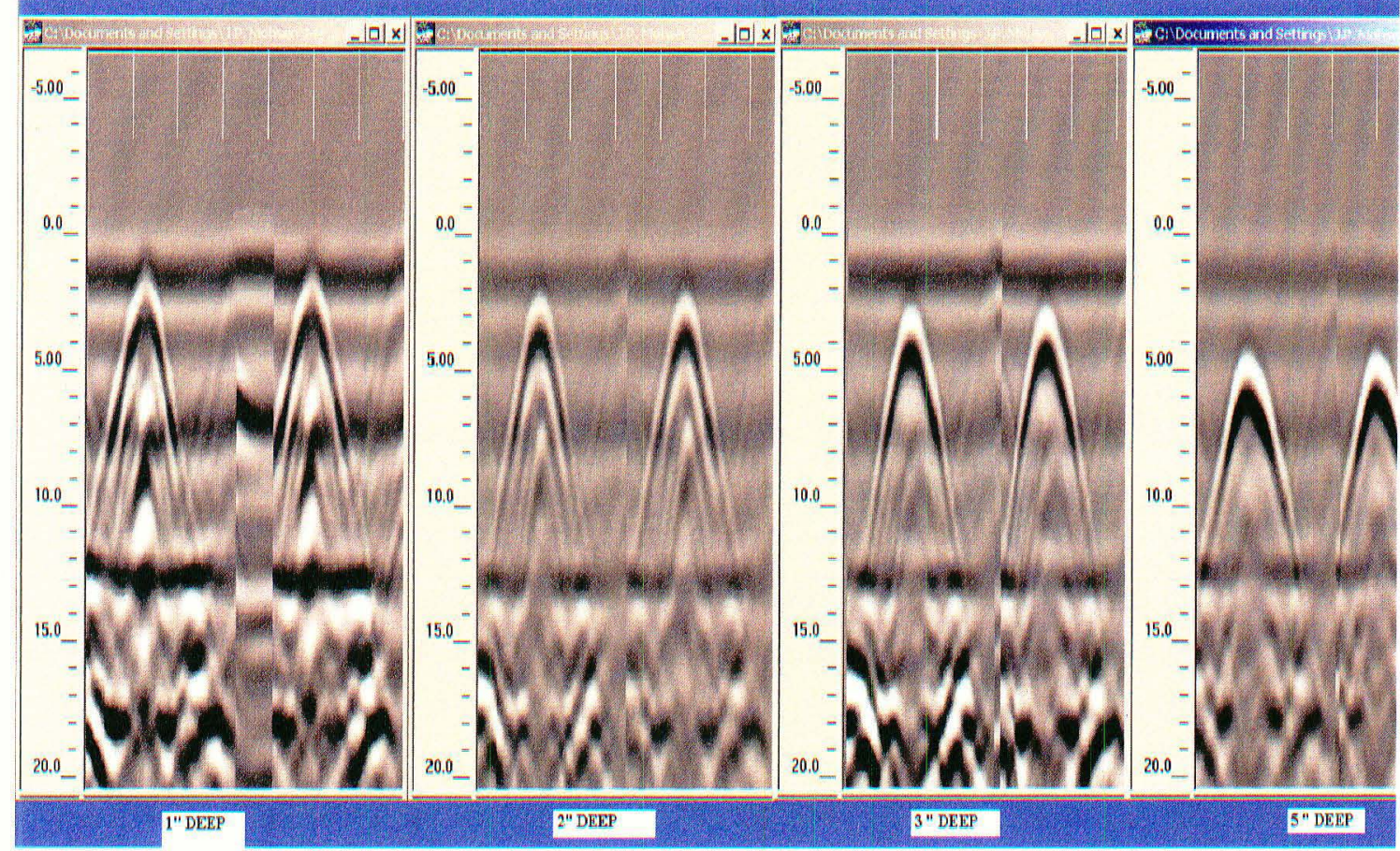

Figure 16: GPR signals of a No 9 steel rebar at different depths 
Fig 17 shows a set of images of different sizes of steel rebars, taken at the same depth using the same operating parameters. The images obtained look similar. The sizes of the steel rebars can not be differentiated visually.

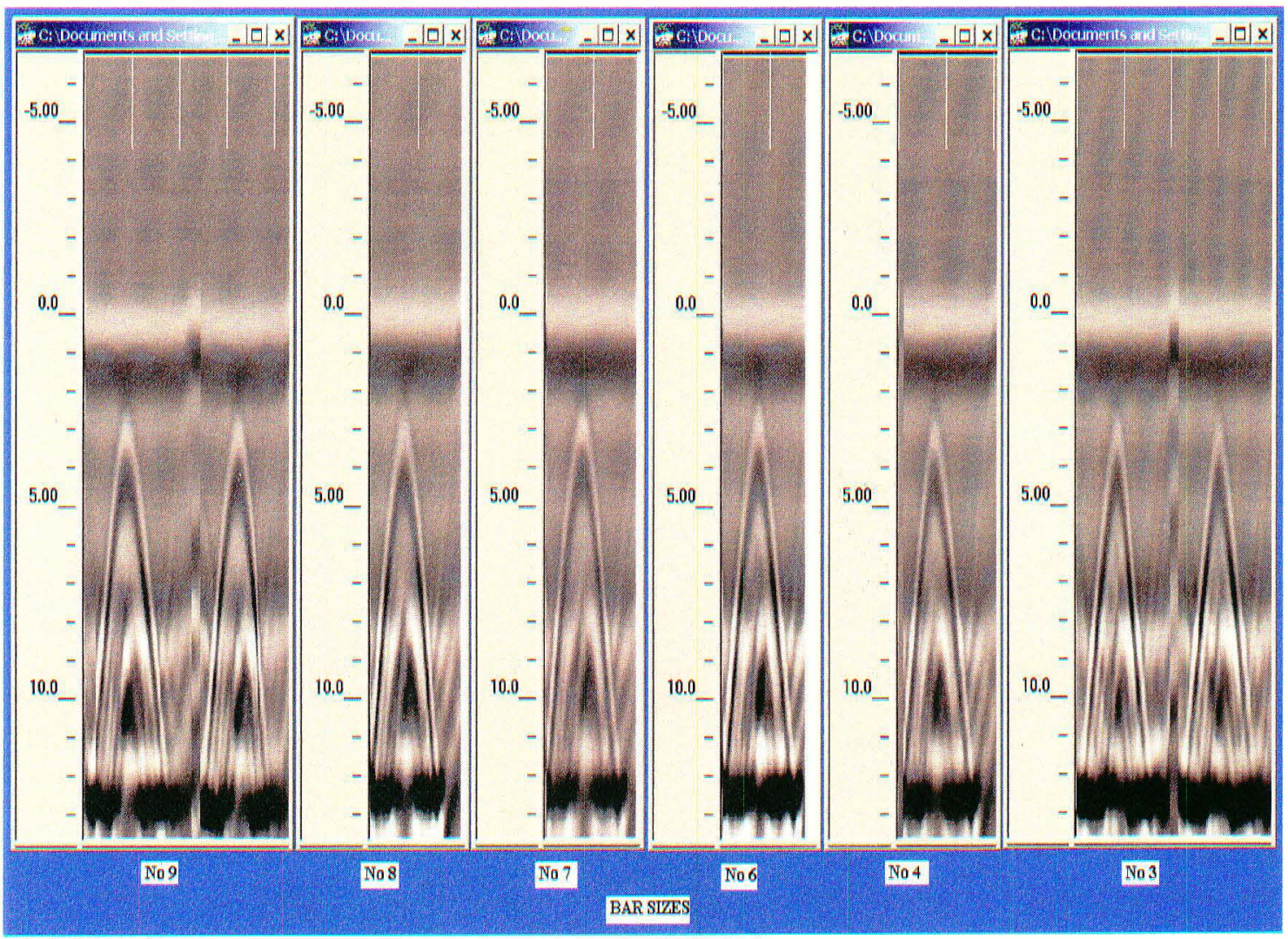

Figure 17: GPR signals of a No 3, No 4, No 6, No 7, No 8, No 9 steel rebars, 3" deep 
The image obtained for a one steel rebar are compared to images of a set of two steel rebars put next to each other horizontally and vertically. The intention is to see if the obtained parabolas can be visually differentiated.

As can be noticed in the figure 18 when a set of two No 8 steel rebars are placed next to each other, they produce similar images to the one produced by one steel rebar. The two set of steel rebars act as if they were one big steel rebar.
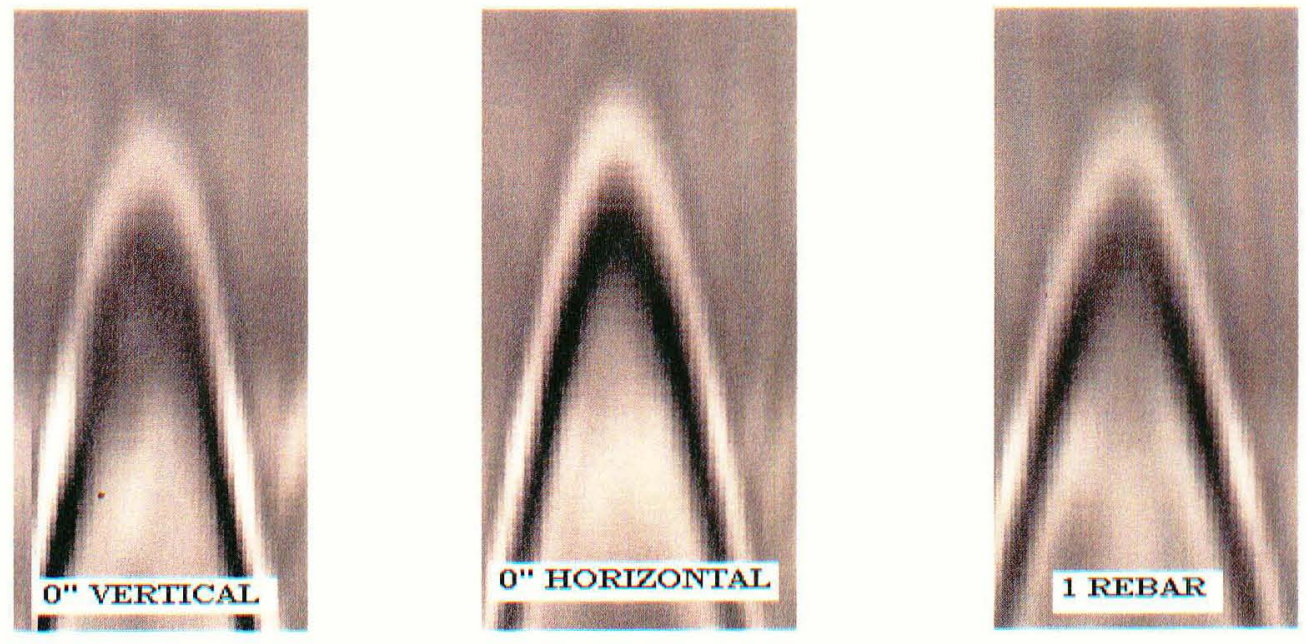

Figure 18: GPR signals of one No 8 steel rebar and two No 8 steel rebars placed next to each other 
The horizontal distance study was done by varying horizontal distances between steel rebars. The different distances are 0", 1", 2", 3" 5" and in some cases $9^{\prime \prime}$.

\section{EQUAL SIZE STEEL REBARS}

Fig 19 shows two No 8 steel rebars placed 1" deep in the sand box. The horizontal distance is varied between 0", 1", 2", 3", 4", 5" and 9" apart. Visual inspection of the figures reveals that as the horizontal distance between two steel rebars increases; the peaks of the GPR signals received will start to further move away from each other. This trend is obvious when the horizontal distance is 2" or more, but also closer inspection of a 1" distance reveals change in the parabola pattern from a 0" distance, as shown by the red and blue ellipses on the figure 20 . 

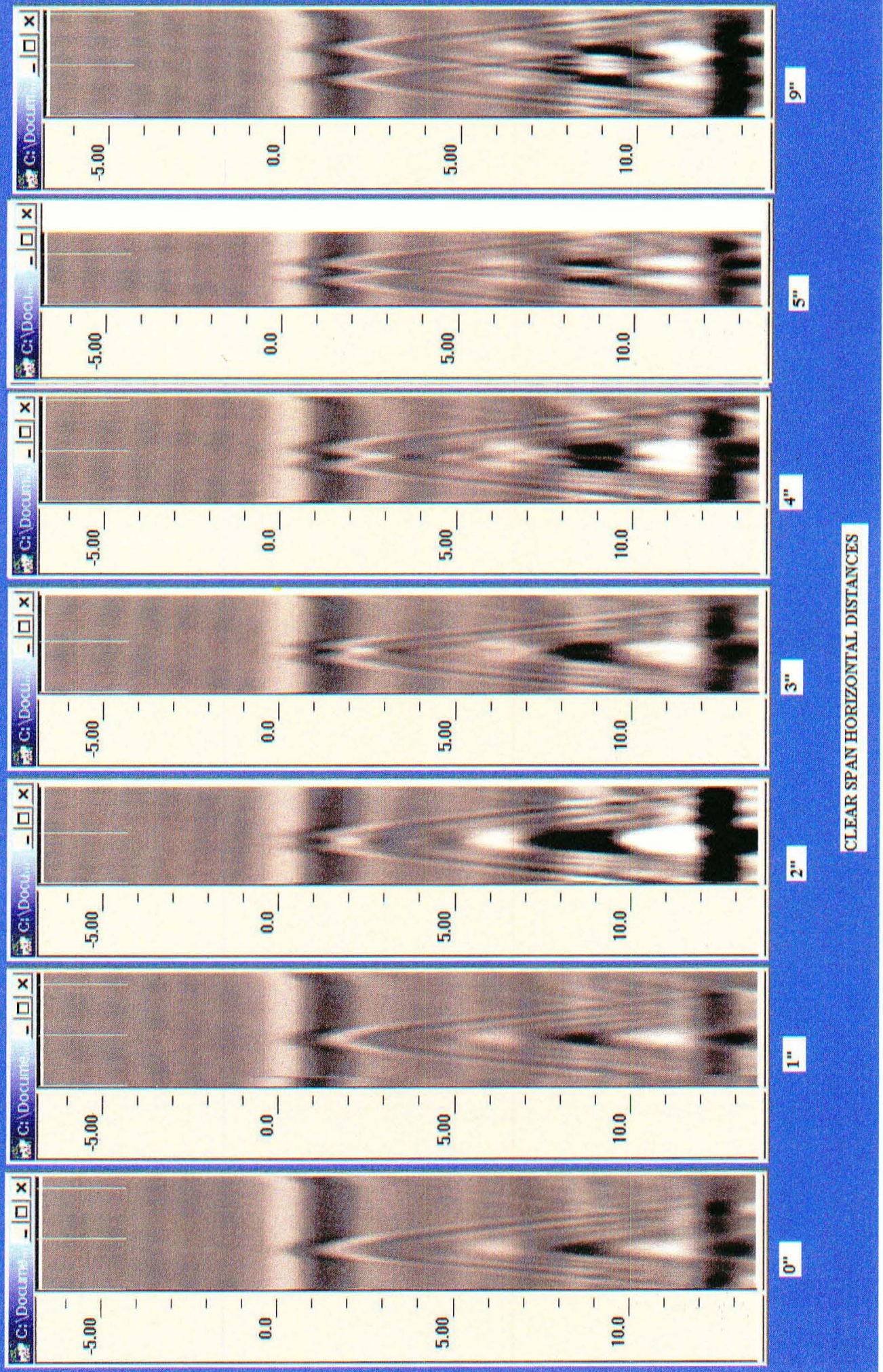

i

Figure 19: Horizontal distance effects between two No 8 steel rebars, 1" deep 

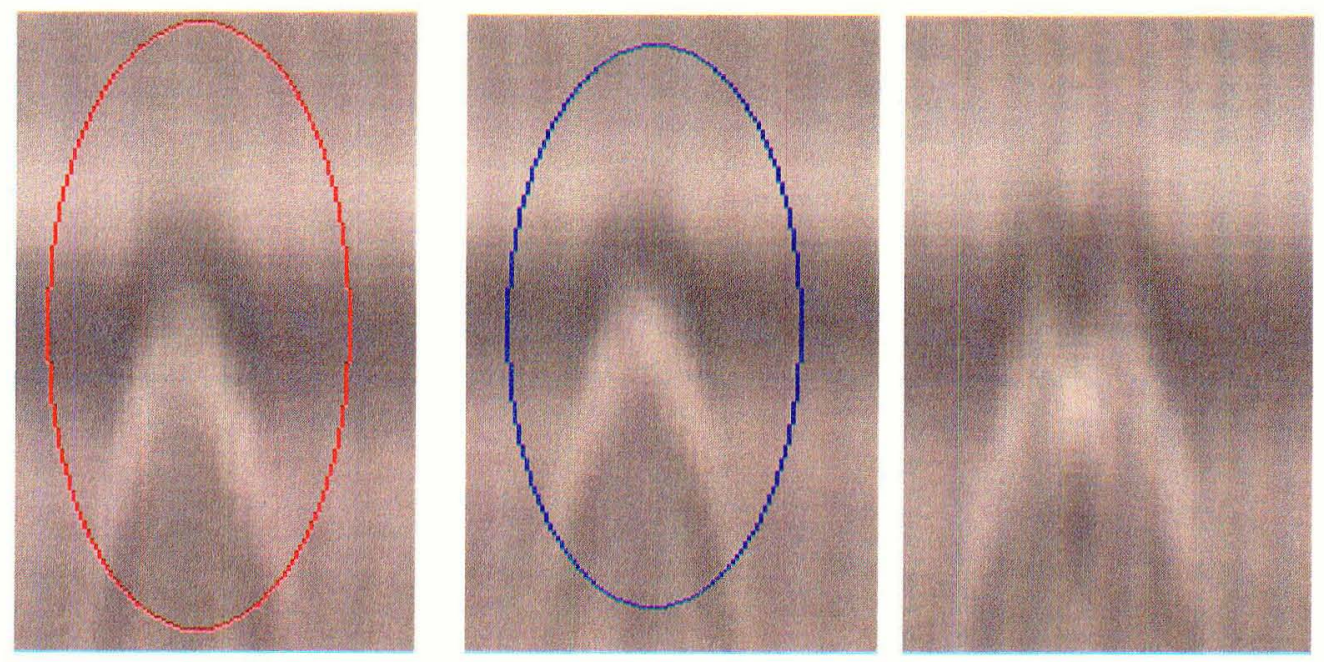

Figure 20: Close up of the parabola peaks of two No 8 at $0^{\prime \prime}, 1^{\prime \prime}, 2^{\prime \prime}$ apart

\section{DIFFERENT SIZE STEEL REBARS}

Two steel rebars one a No9 and the other a No3 were buried in the sand. The peaks of the obtained parabolas are clearer when the horizontal distance is equal to $2^{\prime \prime}$ or is greater, as in the case of equal size rebar. But in this case images of the parabolas when the horizontal distance is equal to $1^{\prime \prime}$ do not change the pattern as much as when the steel rebars were of equal size. 


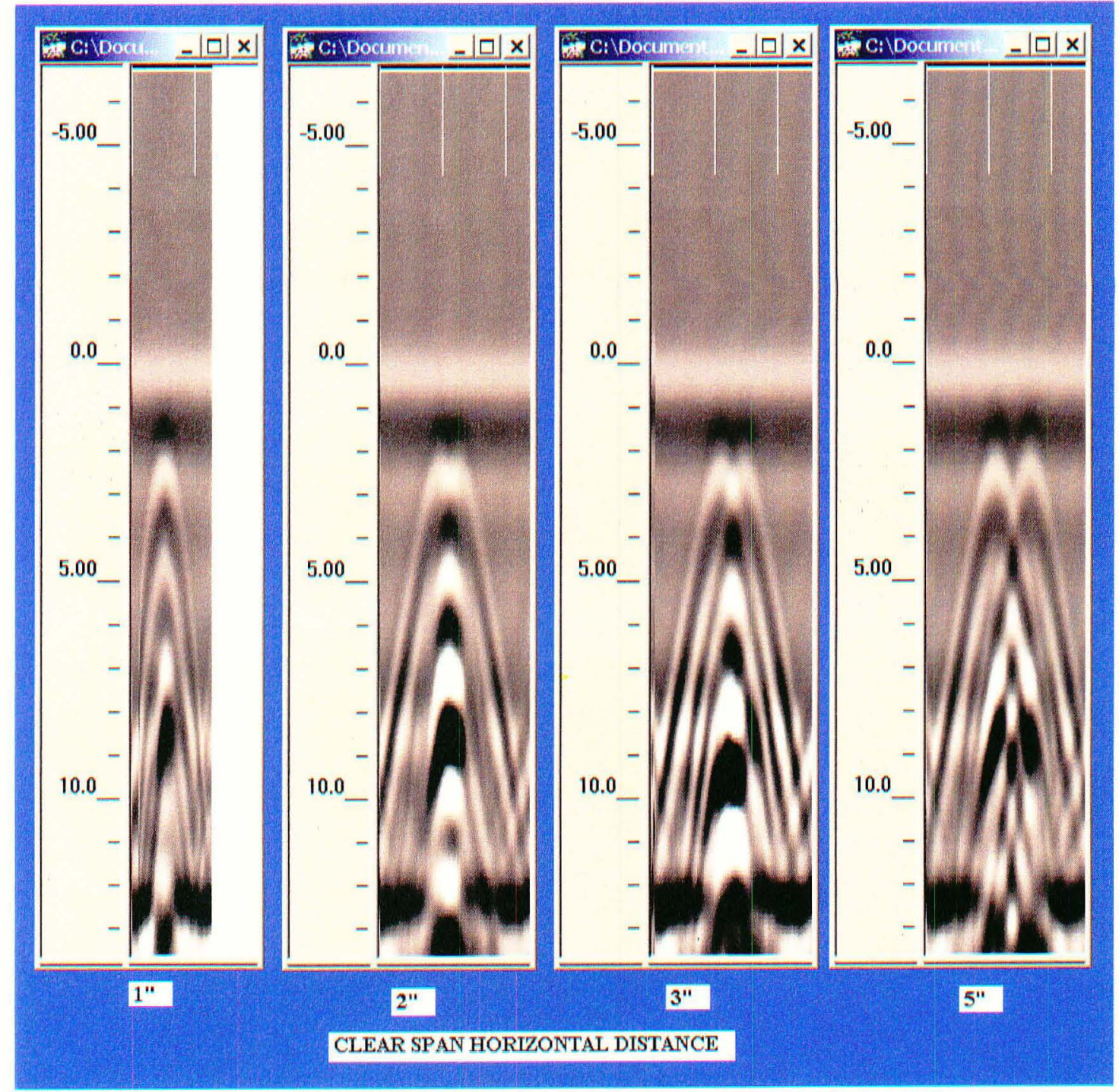

Figure 21: Horizontal distance effects between a No 3 and No 9 steel rebars 


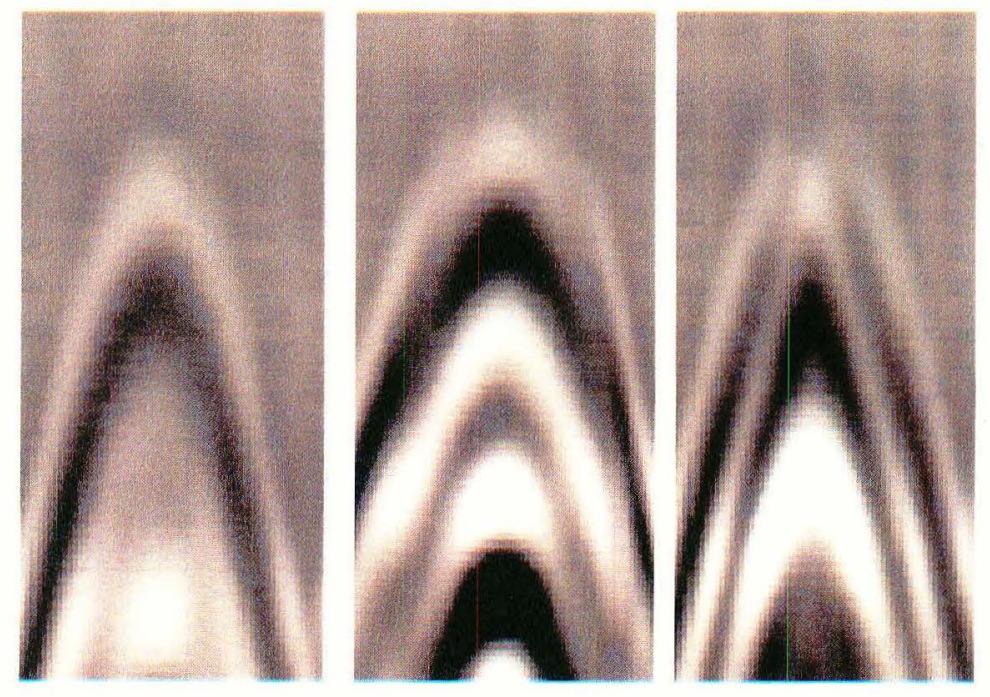

Figure 22: Close up of the parabola peaks of a No 3 and No 9 next to each other

\section{SCAN SPACING EFFECTS}

Increase of the scan spacing parameter increases the details of GPR images displayed. Fig 23 shows the set of images of two steel rebars of equal sizes with $1^{\prime \prime}$ horizontal distance, it is noticed that the details of the two steel rebars parabolas are clearer as the number of scan per inch is increased. 


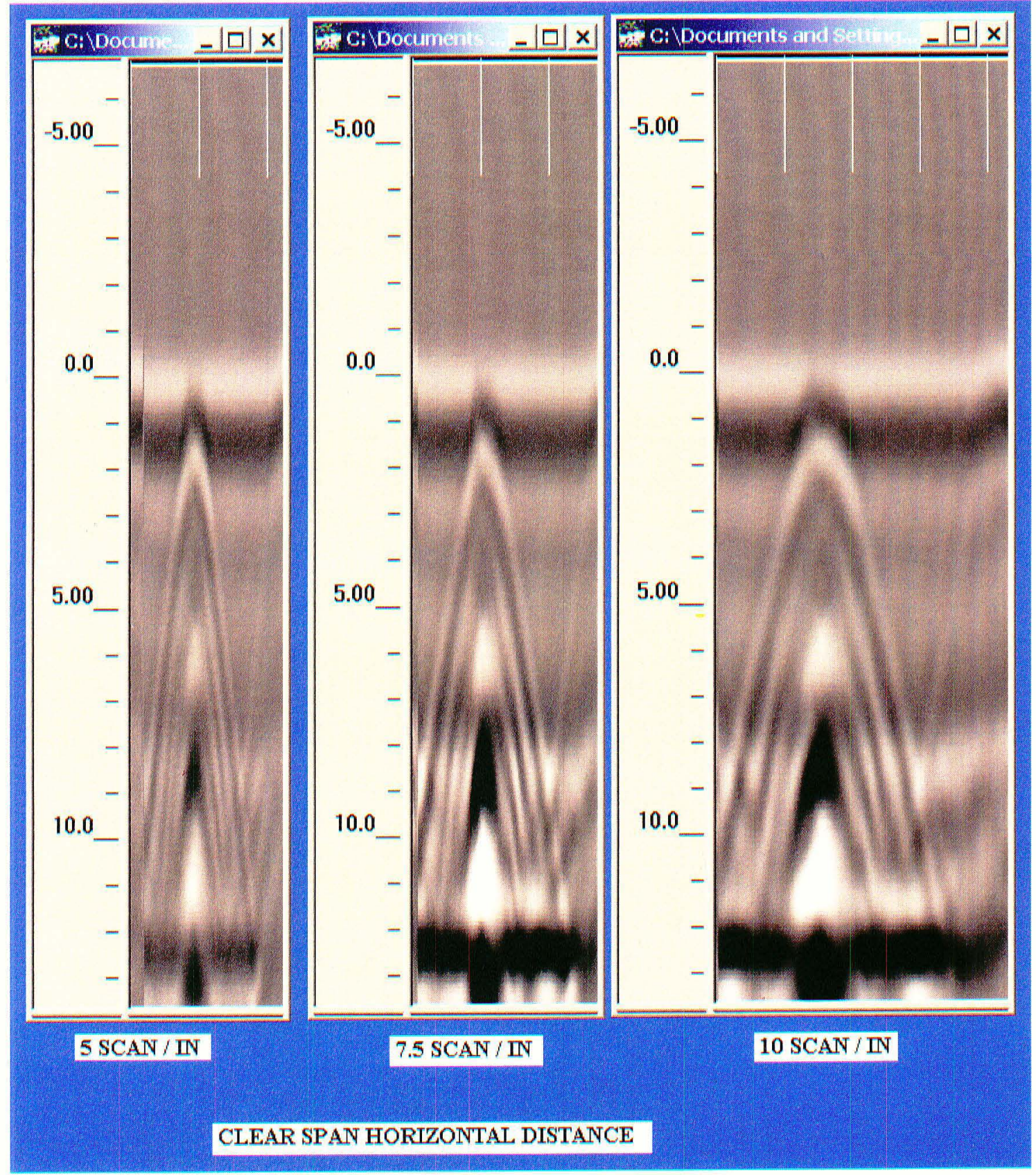

Figure 23: Scan spacing effects on GPR signals of two No 8 steel rebars, 1" deep and 1" apart 


\section{DEPTH OF STEEL REBAR EFFECTS}

This section studies the effects the depth of the parallel rebars has to the signals shown in distinguishing the horizontal distance between them. The images used show a set of two No 8 steel rebars, with 2 " as the horizontal distance between them. The depth of placement varies between $1 ", 2 ", 3 "$ and $5 "$.

As can be seen at the peaks of the parabolas, as the depth of the parallel steel rebars increases, the detection capability of the horizontal distance between steel rebars reduces. The clarity of the twin parabolas reduces as the depth increases, to a point where they are no longer clear, this is at a depth of $5 "$.

This implies that as the depth of the parallel steel rebars increases, they will tend to be assimilated to a one steel rebar. 


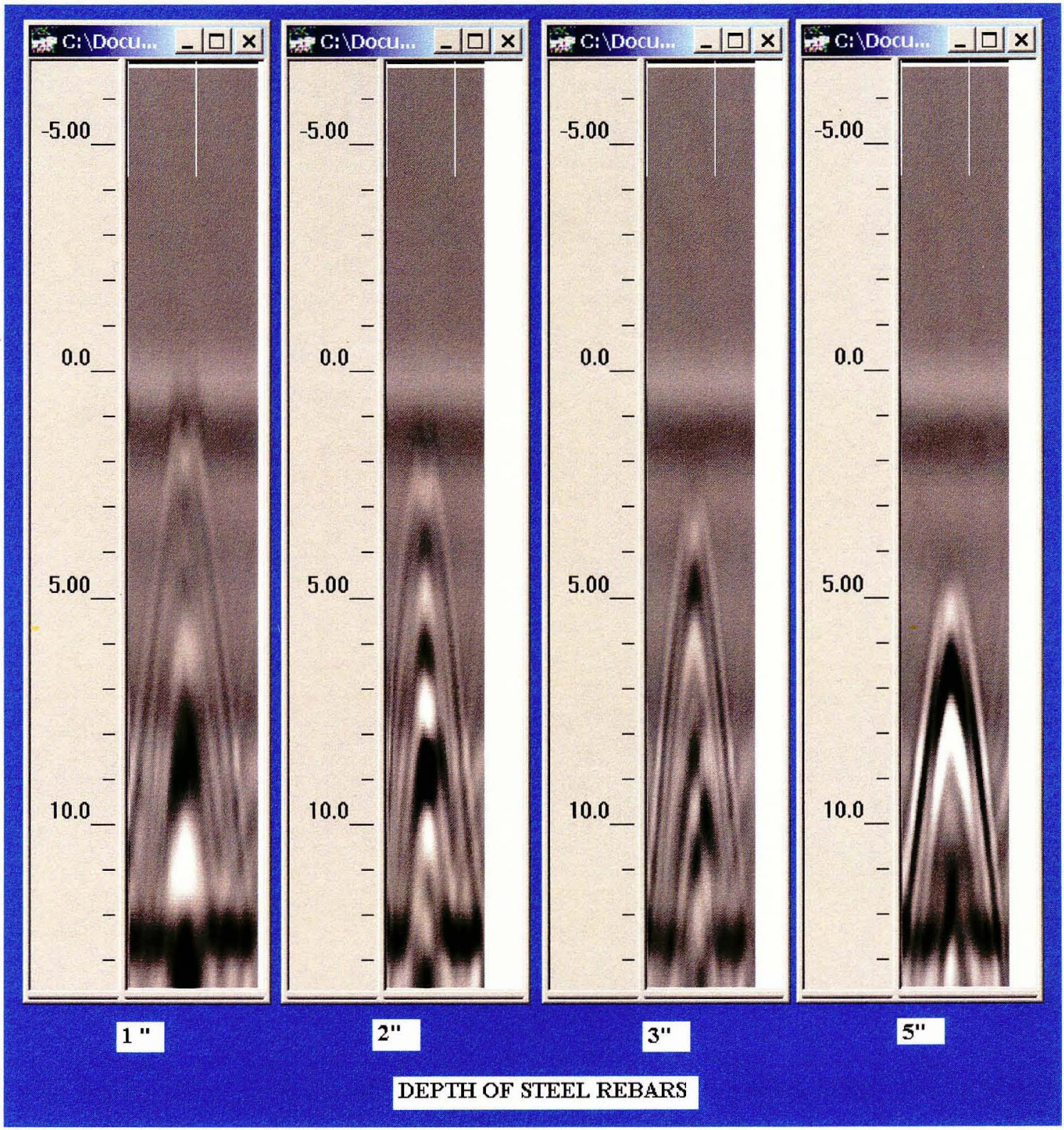

Figure 24: Effect of depth of placement on visibility of two No 8 steel rebars, different placement depths 


\section{EQUAL SIZE STEEL REBARS}

Fig 25 shows a set of two No 8 steel rebars. The vertical distance between them is varied from 1", 2", 3", 4" and 5" apart. The images obtained will show two distinct parabolas one on top of the other. And as the vertical distance is increased, the peaks of the parabolas move further from each other.

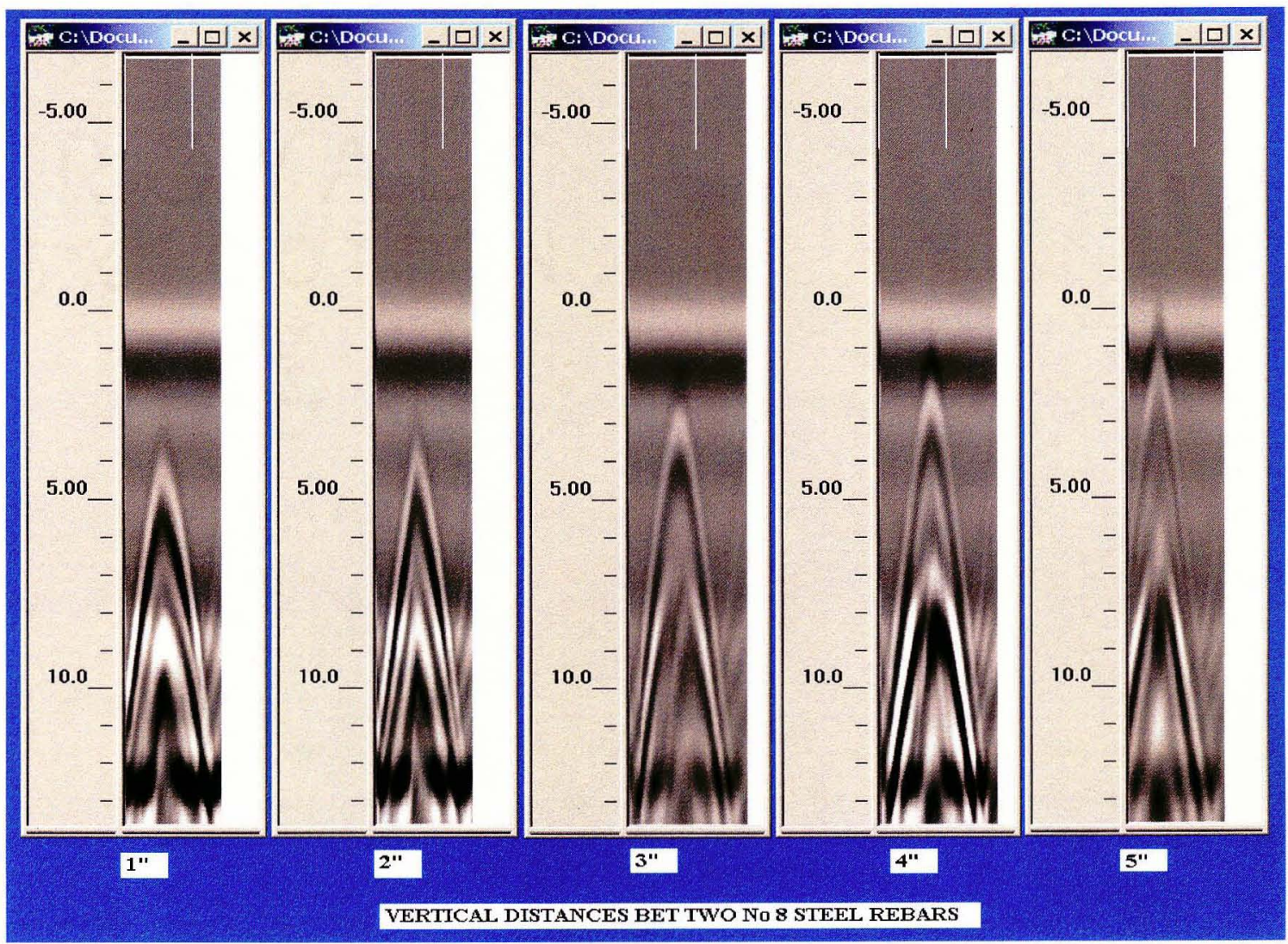

Figure 25: Vertical distance effects between two No 8 steel rebars 


\section{DIFFERENT SIZE STEEL REBARS}

Fig 26 shows two steel rebars of different sizes. The No9 steel rebar is placed at the bottom of the No3 steel rebar. The vertical distance between them is varied from 0", 1", 2", 3", 4" and 5" apart. The images obtained will show two distinct parabolas one on top of the other as in case of equal size steel rebar.

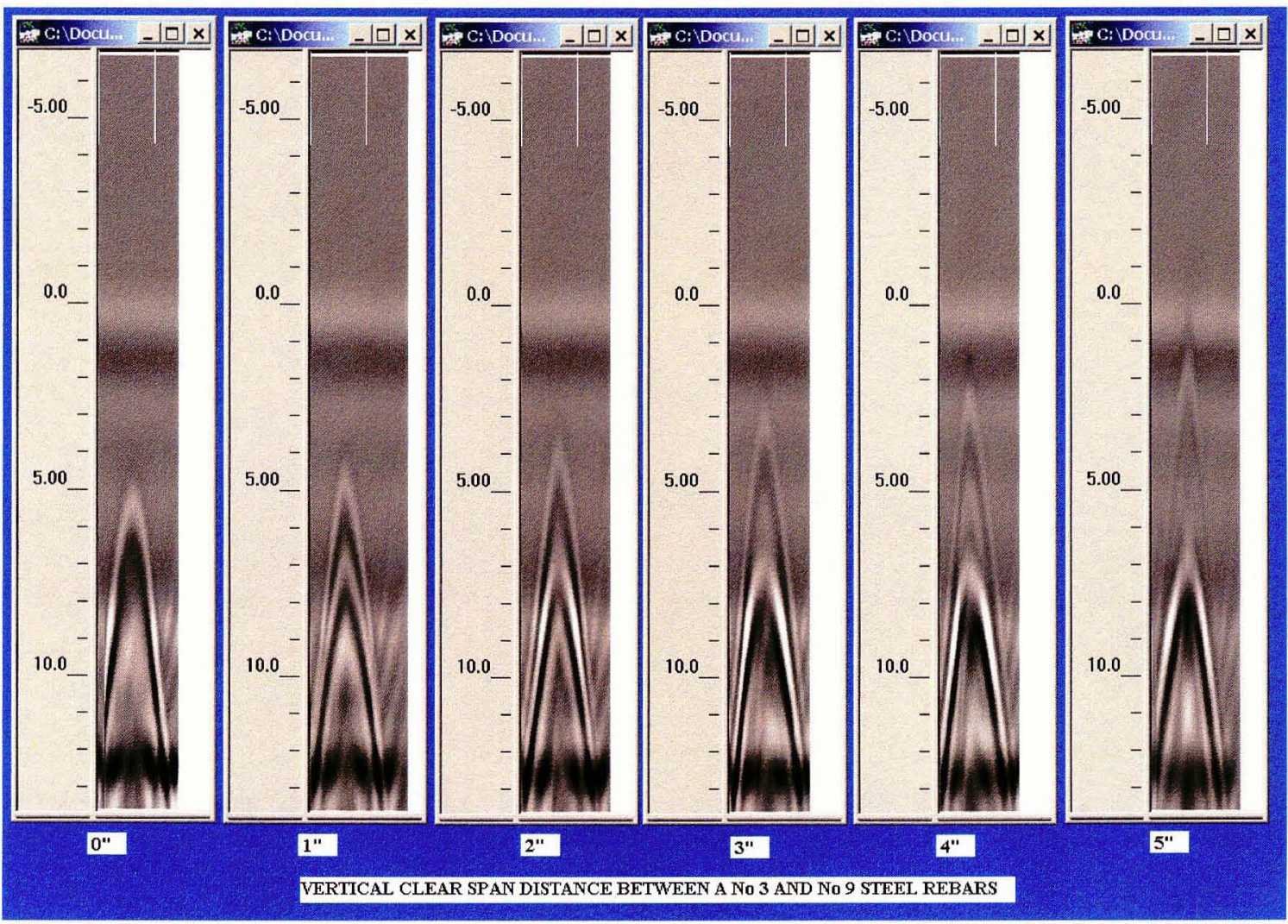

Figure 26: Vertical distance effects between a No 3 and No 9 steel rebars 


\section{CHAP. VIII CONCLUSIONS AND RECOMMENDATIONS}

\section{VIII.1 CONCLUSIONS}

The objective of the research was to provide a library of images that can be used by GPR trainers during GPR technology introduction or that can be used for further studies.

The image library was developed by a series of images collected on different feature configurations by using different operating parameter setups.

Sand medium was used as it has a dielectric constant range that almost mirrors concrete's dielectric constant range.

The results reported are only applicable to the cases studied, in terms of parameters and materials used. 
The following conclusions can be drawn based on the study's objectives:

- A library of GPR images was created and described.

- Comparative studies for different parameter setups were done and the following conclusions reported:

- The scan per unit parameter determines how detailed the survey results images will be. An increase in the scan spacing number increases the details of the survey image.

- Increasing the depth parameter affects the resulting images by raising the features general images higher as the depth of signals shown increase.

- The images obtained from steel rebars located at different depths do not reflect any change in the clarity of the parabola but they show that the steel rebars are not at the same depth. 
- For the size of the steel rebars used, the sizes of the steel rebars can not be differentiated visually.

- A set of two steel rebars placed next to each other, horizontally and vertically produce similar signals to a signal from one steel rebar.

An increase of the horizontal distance between two steel rebars is easily picked up by the antenna when that distance is more than 2 inches for the size of steel rebars used. A 1 inch distance is not easy to pick up between two No 8 size rebars, it is easier to see the horizontal distance between a No9 and a No3 but still more difficult if there is no prior knowledge of the setup.

- As the depth of the parallel steel rebars increases, the detection capability of the horizontal distance between them reduces.

- As vertical distance increase, the signals of the steel rebars move further from each other. In the 
case of two No 8 steel rebars, a horizontal distance of 1 in. is not visible but it is visible between a No 3 on top of a No 9 .

\section{VIII.2 RECOMMENDATIONS AND FUTURE WORK}

The set of GPR images taken are not exhaustive in many regards. Based on the objectives of this experiment, following recommendations and future work are proposed:

- Providing larger display monitors will improve the visual analysis of GPR images.

- Extend the scope of study by using various types of materials and setting up different configurations.

- Extending the study to post-processing parameters effects.

- Extend the study by using other available antennas. 


\section{REFERENCES}

Anatoly Dolgiy, Andrey Dogiy, Vladimir zolotarev (2006). Optimal Radius Estimation For Subsurface Pipes Detected by Ground Penetrating Radar. Proceedings on the $11^{\text {th }}$ International Conference on Ground Penetrating Radar (2006).

Angelo Liseno, Rocco Pierri, raffaele Solimene, Fransesco Soldovieri (2004). Shape Reconstitution Algorithm for Buried objects and Cavities. Proceedings on the $10^{\text {th }}$ International Conference on Ground Penetrating Radar (2004).

Antonio Giannopoulos, Paul Macintyre, Scott Rodgers and Mike C. Forde. (1998). GPR Detection of Voids in Post Tensioned Concrete Bridge Beams. University of Edinburg, School of Civil and Environmental Engineering.

Barry J. Allred, Norman R. Fausey, Chi-Chih Chen, Leon Peters Jr., Hyoung-Sun Youn (2004). GPR Detection of Drainage Pipes in Farmlands. Proceedings on the $10^{\text {th }}$ International Conference on Ground Penetrating Radar (2004).

Caner Ozdemir and Hao Ling (2005). An Experimental Investigation of Buried-objects Imaging in a Homogeneous Medium Using Synthetic-Aperture Radar Concepts. Microwave and Optical Technology Letters Vol. 48, No. 6, June 2006.

Ch. Maierhofer, Th. Kind (2002). Application of Impulse Radar for Non-Destructive Investigation of Concrete Structures. Proceedings on the $9^{\text {th }}$ International Conference on Ground Penetrating Radar (2002). 
Dana Hughes and Reza Zoughi (2005). A Novel Method for Determination of Dielectric properties of Materials Using a Combined Embedded Modulated Scattering and Near-Field Microwave Techniques, Part 1: Forward Model, IEEE Transactions on Instrumentation and Measurement, Vol. 54, No. 6, Dec. 2005.

Dana Hughes and Reza Zoughi (2005). A Novel Method for Determination of Dielectric properties of Materials Using a Combined Embedded Modulated Scattering and Near-Field Microwave Techniques, Part 2: Dielectric Property Recalculation. IEEE Transactions on Instrumentation and Measurement, Vol. 54, No. 6, Dec. 2005.

Egil S. Eide and Jens F. Hjelmstad (2001). 3D Utility Mapping Using Electronically Scanned Antenna Array.

Guido Manacorda, Howard Scott, paul D. Loach, John J. Kazik, Dave Pinchbeck, Meinolf Remeil, JeanPierre Capdevielle, Pascal-Fabien Fournier (2004). The Europian GIGA Project. Proceedings on the $10^{\text {th }}$ International Conference on Ground Penetrating Radar (2004).

GSSI Handbook for RADAR Inspection of Concrete, 2005. Geophysical Survey system, Inc.

James A Doolittle, Fred E. Minzenmayer, Sharon W. Waltaman and Ellis C. Benham. (June 2003): GPR soil suitability maps.

Jeffrey J. Daniels (2000). Ground Penetrating Radar Fundamentals. Prepared as an appendix to a report to the USEPA Region V, Nov. 25, 2000.

Jeffrey L. Lee and Kenneth H. Stokoe, II (2005). Improved Testing speed of the Rolling Dynamic Deflectometer: A Summary. Center for Transprotation Research. UT at Austin.

Jing Li, Huichun Xing, Xuemin Chen, Y. Sun, Richard Liu, Hua Chen, ED Oshinski, Moon Won, german Claros (2004). Extracting Rebar's reflection from 
Measured GPR Data. Proceedings on the $10^{\text {th }}$ International Conference on Ground Penetrating Radar (2004).

Handbook of Non-destructive Testing of Concrete, Second edition; edited by V.M. Malhotra and N. J. Carino.

Melten Ballan, Fikret Gurgen(2000). 2D Ground Penetrating Radar Data Identification. Proceeding of SPIE-The International Society for Optical Engineering, Vol 4084, Pages: 630-634.

Phong M. Luong (2000). Nondestructive Damage Evaluation of Reinforced Concrete Structure Using Infrared Thermography. Proceedings of SPIE- The International Society for Optical Engineering. Pages: $98-107$.

R. Evans, M. Frost, M. Stonecliffe-Jones, N. Dixon (2006). Ground Penetrating Radar Investigations for Urban Roads. Procedings of the Institution of Civil Engineers Municipal Engineer 159 June 2006, Issue ME2, Page 105-111.

RADAN 6.5 User's Manual, (2005). Geophysical Survey System, Inc.

Ralf Birken, Douglas E. Miller, Maclyn Burns, Paul Albats, Robert Casadonte, Ross Deming, Tony Derubeis, Thorkild Hansen and Michael Oristaglio. (2001). Efficient Large-Scale Underground Utility Mapping in New York City Using a Multi-Channel Ground Penetrating Imaging Radar System.

Richard Yelf, Waleed Al-Nuaimy (2004). Classification System for GPR Parameters. Proceedings on the $10^{\text {th }}$ International Conference on Ground Penetrating Radar (2004).

Rolf Gerber, Peter Felix-Henningsen, Christina Salat, Andreas Junge (2004). Investigation of the GPR reflection Pattern for Shallow Depths on a Test Site. Proceedings on the $10^{\text {th }}$ International Conference on Ground Penetrating Radar (2004). 
S. Shihab, W. Al-Nuaimy, A. Eriksen (2004). Radius Estimation for Subsurface Cylindrical Objects Detected by Ground Penetrating Radar. Proceedings on the $10^{\text {th }}$ International Conference on Ground Penetrating Radar (2004).

S. Shihab and W. Al-Nuaimy (2006). Shape Descriptors for Hyperbolic GPR Signature Distrimition. Proceedings on the $11^{\text {th }}$ International Conference on Ground Penetrating Radar (2006).

S. Shihab and W. Al-Nuaimy (2006). Hyperbola Fitter for Characterisation of Cylindrical Targets in GPR Data. Proceedings on the $11^{\text {th }}$ International Conference on Ground Penetrating Radar (2006).

TerraSIRch SIR System-3000 User's Manual, 2004. Geophysical Survey Systems, Inc.

Van Den Bosch I, Vander Vorst A., Lambot S. (2004). Buried Target Signature Extraction from Ground Penetrating Radar Signal Based on the Equivalence Principle. Proceedings on the $10^{\text {th }}$ International Conference on Ground Penetrating Radar (2004).

Will Clark, Brian Burns, James Ralston, Elvis Dieguez (2004). The Measured Effects of Soils on Ground Penetrating Radar Antenna Resolution. Proceedings on the $10^{\text {th }}$ International Conference on Ground Penetrating Radar (2004).

Zhiong Zhao (2005). "Application of Ground Penetrating Radar to Locate Subsurface Voids in Urban Areas." Phd dissertation. 


\section{APPENDIX}

\section{Bibliography}

A.M. Thomas, N. Metje, S.D.F. Rogers and D.N. Chapman (2006). Ground Penetrating Radar Interpretation as a Function of Soils response Complexity in Utility Mapping. Proceedings on the $11^{\text {th }}$ International Conference on Ground Penetrating Radar (2006).

Benedikt Oswald, Kurt Roth, Peter Bastian (2004). Electromagnetic Full Wave Analysis of Ground Penetrating Radar. Proceedings on the $10^{\text {th }}$ International Conference on Ground Penetrating Radar (2004).

D.M. Maurya, B. Goyal, A.K. Patidar, N. Mulchandani, M.G. Thakkar, L.S. Chamyal (2006). Ground Penetrating Radar Imaging of Two Large Sand Blow Craters Related to the 2001 Bhuj Earthquake, kachchh, West India. Journal of Applied geophysics 60 (2006) pages: 142-152.

Demet S. Armagan Sahinkaya, Ahmet Serdar, Turk, Bulent Sen, Sevinc Aydinlik Bechteler (2004). An Innovative GPR System Design: detection of Cylindrical Dielectrical objects. Proceedings on the $10^{\text {th }}$ International Conference on Ground Penetrating Radar (2004).

Doolittle, James A. (2003). Ground Penetrating Radar Soil Suitability Maps. Journal of Environmental \& Engineering geophyisics 2003, Pages: 49-58. 
Dr Alexander, SD Kohn, WP Grogan (1989).

Nondestructive Testing Techniques and Evaluation for Airfield Pavements. ASTM Special Technical publication, pages 502-524.

Dryver Huston, Peter Fuhr, Ken Maser and William Weedon (2002). Nondestructive testing of Reinforced Concrete Bridges Using Radar Imaging Techniques. Report prepared for The New England Transportation Consortium.

Eiji Sakurada, masaharu Inagaki (2002). GPR Rapid Survey system For Small Diameter Tunnels. Proceedings to the $9^{\text {th }}$ International Conference on Ground Penetrating Radar (2002).

Fan-nian Kong (2000). Choice of Antenna Type and Frequency Range for Testing Concrete Structures. Norwegian Geotechnical Institute.

G. Triltzsch, H.M. Braun, Y. Krellman, A. Prugger, W.G. Maybee, S.Maloney, P.K. Kaiser. Stepped Frequency GPR Field Trials in Potash Mines. Proceedings on the $10^{\text {th }}$ International Conference on Ground Penetrating Radar (2004).

J.P.Mohsen, T.D.Rockaway, D.K.Gupta (2006). Test Field Design and development for GPR Techniques for Infrastructure Asset Identification. Proceedings on the $11^{\text {th }}$ International Conference on Ground Penetrating Radar (2006).

Jeff R. Brown and H.R. Hamilton III (2003). NDE of Reinforced Concrete strengthened with FiberReinforced Polymer Composite Using Infrared Thermography.

Lawrence B. Conyers (2004). Moisture and Soil Difference as Related to the Spatial Accuracy of GPR Amplitude Maps at two Archaeological Test Sites. Proceedings on the $10^{\text {th }}$ International Conference on Ground Penetrating Radar (2004). 
M. R. Shaw, S G Millard, T C K Molyneaux, J H Bungey, M J Taylor (2002). Location of Steel

Reinforcement in Concrete Using Ground Penetrating Radar and Neural Networks.

S.R. Pennock, M.A. Redfern (2006). Multihead Configuartion for Ground penetrating Radar and Depth Determination. Proceedings to the $11^{\text {th }}$ International Conference on Ground Penetrating Radar (2002).

S. Shihab, W. Al-Nuaimy, A. Eriksen (2004). Radius Estimation for Subsurface Cylindrical objects Detected by Ground Penetrating Radar. Proceedings on the $10^{\text {th }}$ International Conference on Ground Penetrating Radar (2004).

Vinayak M. Deshpande and Manohar D. Deshpande (2005). Study of Electromagetic Wave Propagation Through Dielectric Slab Doped Randomly with Thin Metallic Wires Using Finite Element Method. IEEE Microwave and Wireless Components Letters, Vol.15 No. 5 May 2005 .

Yuji Takeshita, Hiroaki Kobayashi, Kazunori Tao, Ichiro Kaihotsu (2004). Measurement of Groundwater Behavior in Sandy Soils Using Surface Ground Penetrating Radar. Proceedings on the $10^{\text {th }}$ International Conference on Ground Penetrating Radar (2004). 


\section{CURRICULUM VITAE}

NAME:

Claudel NISINGIZWE

ADDRESS:

Department of Civil and Environment Engineering

Univerity of Louisville

Louisville KY, 40219

DOB:

Kigali, Rwanda - May 26, 1980

\section{EDUCATION}

\& TRAINING: M.S., Civil Engineering.

Department of Civil and Environment Engineering University of Louisville

$2006-2007$ 
B.S., Civil Engineering and Environmental Technology

KIST, (Rwanda)

$1999-2004$

AWARDS: $\quad$ Best Student Presentation, Third place

2006 HEEP CONFERENCE

Louisville, KY

2006

\section{PROFESSIONAL}

SOCIETIES:

ASCE.

CHI - EPSILON

Golden Key Honor Society member

NSBE

\section{NATIONAL MEETING PRESENTATIONS:}

Poster presentation: Test Field Design and Development for GPR Techniques for Infrastructure Asset Identification. $11^{\text {th }}$

International Conference on GPR 2006

Test Field Design and Development for GPR Techniques and Its application on Transportation. 2006 HEEP Conference 